\title{
Dynamics analysis of a hollow-shaft rotor system with an open crack under model uncertainties
}

\author{
Chao Fu ${ }^{1,2 \dagger}$, Yuandong Xu${ }^{1}$, Yongfeng Yang ${ }^{2}$, Kuan $\mathrm{Lu}^{2}$, Fengshou Gu${ }^{1}$, Andrew Ball ${ }^{1}$ \\ ${ }^{1}$ Centre for Efficiency and Performance Engineering, University of Huddersfield, \\ Queensgate, Huddersfield, HD1 3DH, United Kingdom \\ ${ }^{2}$ Institute of Vibration Engineering, Northwestern Polytechnical University, Xi' an 710072, P.R. China
}

\begin{abstract}
This paper focuses on the vibration behaviors of a hollow-shaft rotor in presence of an open crack under inherent uncertainties. Non-probabilistic interval variables are used to represent the uncertain parameters, which releases the high demands of probabilistic knowledge in traditional methods. In modeling the shaft, local stiffness matrix of the cracked element is derived by using the neutral axis method. The periodic response of the rotor system is solved by combination of the finite element method (FEM) and the harmonic balance method (HBM). A simple mathematical function, termed as the uncertain response surrogate function (URSF), is constructed to estimate the vibration response in various cases where different parametric uncertainties are taken into consideration. To verify the robustness and accuracy of the URSF, the bounds of estimated response are compared with those obtained from the classical methods. Results show that the surrogate function has good accuracy and robustness, providing an effective method and guidance for diagnosing crack in uncertain context.
\end{abstract}

Keywords: hollow-shaft rotor; interval uncertainty; open crack; surrogate function; vibrations

\section{Introduction}

Due to the overload operations of rotors in large rotating machineries, cracks in the rotation shafts often occur. In the recent decades, crack has become a central issue of concerns in the faults of rotor systems, attracting the attention of researchers in many areas [1-5]. Many vibrational characteristics-based crack detection methods have been developed [6-8]. The FEM in combination of the HBM is usually adopted to solve the steady dynamic responses of rotor systems with cracks [9]. A finite element model is constructed for a cracked rotating compressor blade by Zeng et al. [10]. Guo et al. [11] carried out experimental studies on the crack detection of a Jeffcott rotor, which proved the effectiveness of the EMD based method. Chen et al. [12] proposed an analytical method to calculate the mesh stiffness of spur gears and the influences of crack depth were investigated in detail. In the derivation of the global equations of motion for the rotors, it is important to describe the time-varying stiffness matrix of the cracked shaft element accurately [13-14]. The common modeling techniques include the neutral axis method [15] and the fracture mechanics [16]. In the advanced period of the breathing cracks, it is

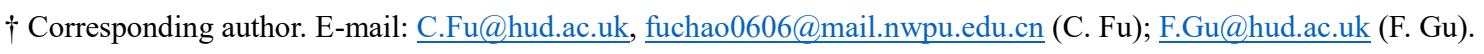


dominated by the open cracks $[17,18]$. Dharmaraju et al. [19] proposed an identification method to estimate the flexibility of the cracked element and the depth using the force and response data. Faverjon and Sinou studied a simply supported beam with an open crack and developed a robust damage identification algorithm [20]. Furthermore, the interactions of two cracks and their influences on the eigenfrequencies and modes of rotor systems were discussed in Refs. [21, 22]. Hollow shafts are commonly used in engineering flexible rotor systems. However, inadequate attention has been paid to the vibrations of cracked hollow-shaft rotor systems. Lu et al. [23, 24] studied the effects of a breathing crack in the hollow shaft of a dual-rotor system and the open crack of a high pressure rotor. These researches provided insightful views of the dynamic behaviors of cracked rotors with both breathing and open cracks, which are helpful for damage diagnosis in industrial context.

Practically, the uncertainties are inherently present [25] in mechanical systems due to wear, properties variations and working condition evolutions. The aforementioned contributions had focused on the deterministic models with constant physical parameters. However, the uncertainty should be taken into consideration for a better understanding of the nonlinear dynamic behaviors of cracked rotor systems. Probabilistic methodologies are widely employed for uncertainty quantifications (UQ) in mechanical systems in premise of sufficient statistical data or the distribution models are known already. In this frame, many specific methods have been developed and the popular ones include the Monte Carlo Simulation (MCS), the perturbation techniques [26], the Neumann method [27] and the polynomial chaos expansion (PCE) [28]. The MCS with enough samples can provide accurate estimations but the convergence rate is rather slow. To alleviate this, Koroishi et al. [29] developed a stochastic FE method for uncertain rotordynamics based on the combination of the MCS and the Latin hypercube sampling technique. Several probabilistic models were adopted for the robust optimization of a flexible rotorbearing system by Ritto et al. [30]. Didier et al. [31, 32] carried out insightful studies of uncertain rotor systems with nonlinearities and stochastic uncertainties using the PCE, which represents the uncertainties by the orthogonal polynomial series based on the Gaussian distribution. Many kinds of faults and non-regular nonlinearities were discussed in [33-36]. Sinou et al. [37] focused on the vibration signatures of a rotor containing a chord crack and it was found that the PCE is valid for random UQ in cracked rotors. The influence of the PCE order on the dynamic response of an asymmetric rotor system was further investigated [38]. Other applications include the unbalance and balancing aspects [39] as well as critical speed analyses [40]. Attention to fuzzy approach was paid in Ref. [41].

Under the circumstances that sufficient information is unavailable to define the distribution models of uncertainties, which is frequently encountered in engineering, the non-probabilistic methodologies can be applied alternatively [42-47]. The interval-based approaches [48-50], which require the bounds of the uncertain parameters only, are the typical ones among them. The orthogonal Chebyshev series based algorithms [42, 48, 49] are transparent, transplantable and non-intrusive. These methods transform the uncertain dynamic equations into a set of deterministic ones without breaking into the original solution process. So far, interval analysis in rotordynamics has not attract adequate attentions, especially in the cases of cracked rotors. Attempt to implement interval calculation in rotors was made by Dimarogonas 
[51]. Ma et al. [52] and Wang et al. [53] used the interval arithmetic and the perturbations for uncertain dynamic characteristic analysis of linear rotor systems. An interval optimization scheme was proposed by Shiau et al. [54] for rotor-bearing systems. Fu et al. formulated the derivative-based interval method [55] and the interval precise integration method [56] to study the steady-state and transient response of an uncertain overhung rotor system. The vibrations of hollow-shaft rotating systems with an open crack and interval uncertainties remain misunderstood, which will be the main content of this study.

The rest of this paper is organized as follows. The FE model containing an open transverse crack is described in Section 2. In Section 3, the modeling of the cracked element using the neutral axis theory and the governing equations of motion are presented. The formulation of the non-intrusive URSF is detailed in Section 4 based on the Chebyshev collocations. Section 5 mainly presents the numerical results and case studies as well as comparisons with the MCS or the scanning method. Conclusion remarks are summarized in Section 6.

\section{The FE rotor model with an open crack in the hollow shaft}

A sixteen-element rotor model is considered and its schematic diagram is shown in Fig. 1. The hollow shaft is modeled with Timoshenko beam elements where $D_{1}$ and $D_{2}$ are the inner and outer diameters, respectively. For each node on the rotor, four degrees-of-freedom (two rotations and two lateral displacements) are included with the torsional and axial vibrations not being considered. Two rigid disks are assembled at nodes 4 and 14 while the mass unbalance is only considered on the first one. An open crack is present in the hollow shaft as marked in Fig. 1. Isotropic bearings are adopted in the model, which have the same values of damping and stiffness in two orthogonal directions. Table 1 gives the physical parameters of the rotor system. The slight mass unbalance is given in the form of $m_{e} d$ with an initial unbalance phase angle 0 rad. The first two forward critical speeds of the rotor without crack are $599.1 \mathrm{rad} / \mathrm{s}$ and $3280 \mathrm{rad} / \mathrm{s}$.

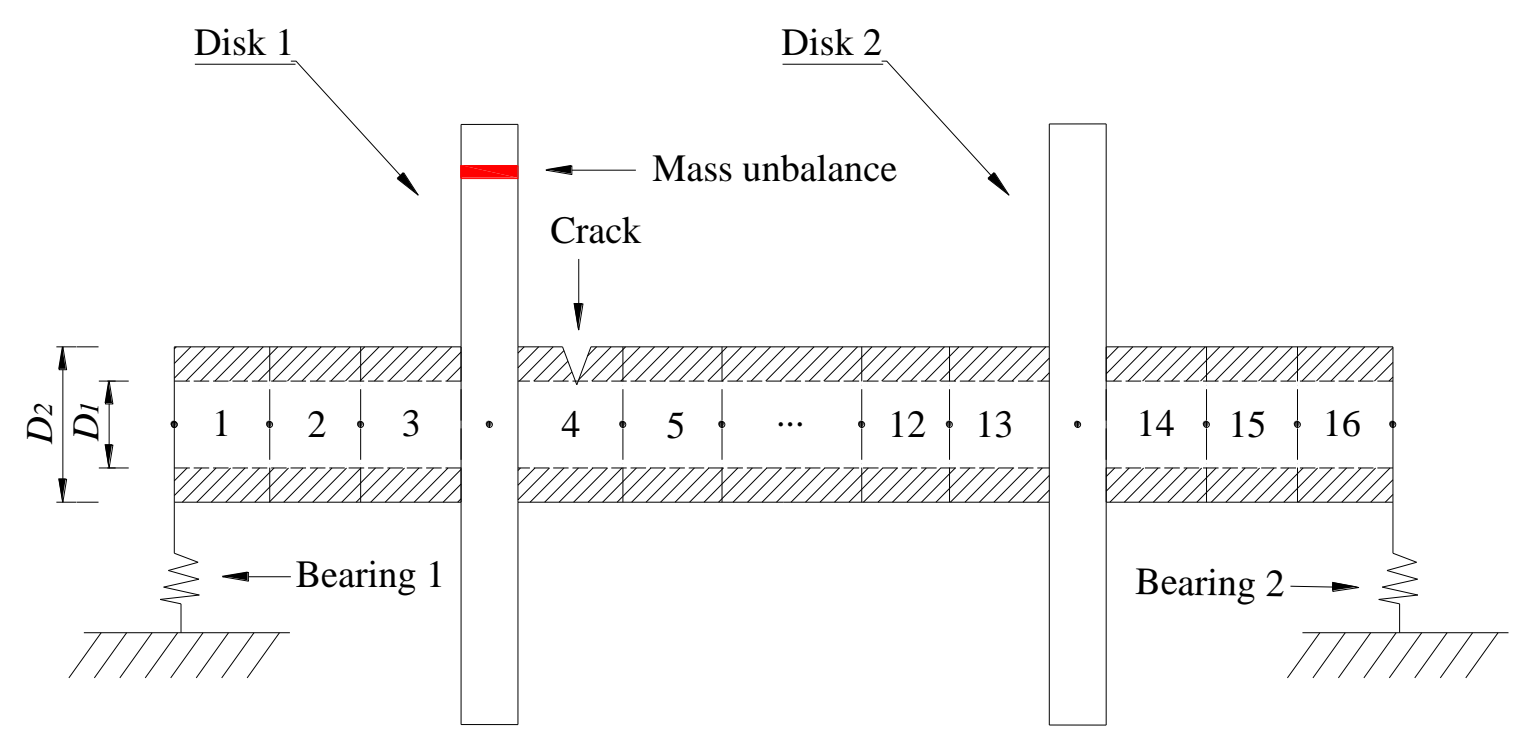

Fig.1. The FE model of the rotor system. 
Table 1

Physical parameters of the system.

\begin{tabular}{llll}
\hline Description & Value & Description & Value \\
\hline Length of shaft, $L$ & $0.96 \mathrm{~m}$ & Young's modulus, $E$ & $2.1 \times 10^{11} \mathrm{~N} \cdot \mathrm{m}^{-2}$ \\
Inner diameter of shaft, $D_{1}$ & $0.03 \mathrm{~m}$ & Bearing damping $\left(c_{x x}=c_{y y}\right)$ & $800 \mathrm{~N} \cdot \mathrm{s} \cdot \mathrm{m}^{-1}$ \\
Outer diameter of shaft, $D_{2}$ & $0.048 \mathrm{~m}$ & Bearing stiffness $\left(k_{x x}=k_{y y}\right)$ & $1 \times 10^{7} \mathrm{~N} \cdot \mathrm{m}^{-1}$ \\
Disk mass, $m_{d}$ & $2.4 \mathrm{~kg}$ & Mass unbalance, $m_{e} d$ & $1 \times 10^{-5} \mathrm{~kg} \cdot \mathrm{m}$ \\
Radius of the disk, $R_{0}$ & $0.08 \mathrm{~m}$ & Density, $\rho$ & $7800 \mathrm{~kg} \cdot \mathrm{m}^{-3}$ \\
Initial unbalance angle, $\phi$ & $0 \mathrm{rad}$ & Poisson's ratio, $v$ & 0.3 \\
\hline
\end{tabular}

\section{Derivation of the governing motion equations and solutions}

The rotor system can be modeled by the FEM in a standard way except for the cracked element. The neutral axis method was previously formulated to calculate the unsymmetrical time-varying stiffness matrix for solid shaft element with an open crack $[8,15]$. For the case of hollow shaft, the crack should be treated separately considering whether the crack has penetrated into the inner cross-section [23, 24]. The geometry evolution of the cracked element cross-section with time is illustrated in Fig. 2, where the shadowed segments denote the uncracked portions. The fixed centroid of the cross-section is $O$, if no crack appears. Due to the presence of crack, the new centroid $O^{\prime}$ is time variant with $e$ being the distance of the two centroids. In Fig. 2, four sets of coordinates are placed: the fixed global coordinates $O X Y$ and the rotating coordinates $O x y$; the new fixed coordinates $O^{\prime} \bar{X} \bar{Y}$ and the related rotating coordinates $O^{\prime} \overline{x y}$. Their axial relationships can be identified from Fig. 2. The initial phase angle of the crack is $\varphi$ regarding to the negative axis $O Y$. In Fig. 2(a), $h$ represents the depth and $\alpha$ is the crack angle. After time $t$ from the initial state, the cross-section is rotated to a new position as shown in Fig. 2(b) where $\Omega$ is the rotating speed. 
(a)

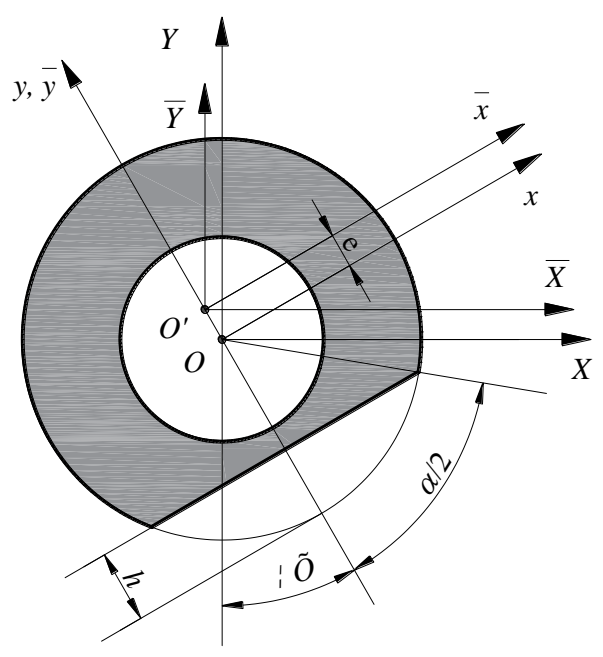

(b)

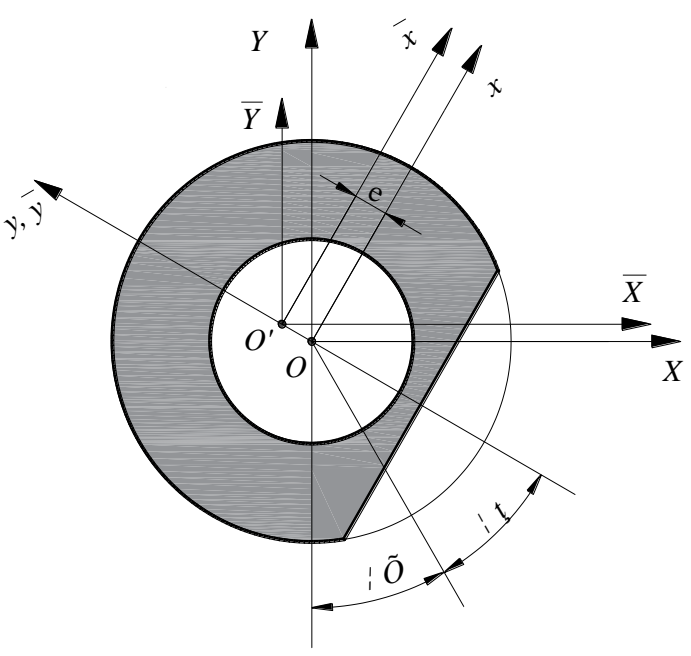

Fig.2. Evolution diagram of the cracked shaft cross-section: (a) initial state, (b) rotation state.

In the following calculation, the initial crack angle $\varphi$ is set to zero. For shallow crack as shown in Fig. 2, the centroid deviation distance can be given by

$$
e=\frac{2}{A_{\text {rest }}} \int_{-(R-h)}^{R} \sqrt{R^{2}-y^{2}} y d y
$$

where $A_{\text {rest }}$ represents the area of the intact region and $R$ is the outer radius of the shaft element. In order to calculate the rest area, the cross-section is regarded as the difference of the outer section and in the inner section as $A_{\text {rest }}=A_{s}^{(1)}-A_{s}^{(2)}$ where $A_{s}^{(1)}$ and $A_{s}^{(2)}$ are the areas of the uncracked segment of the two sections, respectively. They are calculated as [24]

$$
\begin{gathered}
A_{s}^{(1)}=R^{2}[\pi-\alpha / 2+(1-\mu) \gamma] \\
A_{s}^{(2)}=\pi r^{2}
\end{gathered}
$$

in which $\mu$ is the non-dimensional crack depth and can be calculated as $\mu=h / R$. Other notations are the crack size angle $\alpha=2 \arccos (1-\mu)$, the radius of the inner section $r$ and $\gamma=\sqrt{2 \mu(1-\mu)}$. For deep cracks where the inner section is also cracked, the expressions for the quantities of the outer section stay the same while for the inner section the area of the uncracked portion should be expressed as

$$
A_{s}^{(2)}=\pi r^{2}-\alpha_{1} r^{2} / 2+\sqrt{r^{2}-(R-h)^{2}}(R-h)
$$

where $\alpha_{1}=2 \arccos [(R-h) / r]$. The corresponding expression for Eq. (1) is changed to

$$
e=\frac{2}{A_{r e s t}}\left(\int_{-(R-h)}^{R} \sqrt{R^{2}-y^{2}} y d y-\int_{-(R-h)}^{r} \sqrt{r^{2}-y^{2}} y d y\right)
$$

The moments of inertia of the cracked cross section in the rotating frame $O^{\prime} \overline{x y}$ are constant. They are time-variant quantities when projected to the fixed coordinate system $O^{\prime} \bar{X} \bar{Y}$. The detailed derivations can be found in [15]. The expressions can be given as 


$$
\begin{gathered}
I_{\bar{X}}(t)=\frac{I_{\bar{x}}+I_{\bar{y}}}{2}+\frac{I_{\bar{x}}-I_{\bar{y}}}{2} \cos (2 \Omega t)+I_{\overline{x y}} \sin (2 \Omega t) \\
I_{\bar{Y}}(t)=\frac{I_{\bar{x}}+I_{\bar{y}}}{2}-\frac{I_{\bar{x}}-I_{\bar{y}}}{2} \cos (2 \Omega t)-I_{\overline{x y}} \sin (2 \Omega t) \\
I_{\bar{X} \bar{Y}}(t)=-\frac{I_{\bar{x}}-I_{\bar{y}}}{2} \sin (2 \Omega t)+I_{\overline{x y}} \cos (2 \Omega t)
\end{gathered}
$$

where the subscripts designate the corresponding area moments of inertia with respect to the specified axes. Specially, due to the symmetry of the cross-section about the rotating $y$-related axis, we have $I_{\overline{x y}}=0$. The $I_{\bar{x}}$ and $I_{\bar{y}}$ are derived as a combination of the two cross-sections

$$
\begin{gathered}
I_{\bar{x}}=I_{x}-A_{s}^{(1)} e^{2}=I_{x}^{(1)}-I_{x}^{(2)}-A_{s}^{(1)} e^{2} \\
I_{\bar{y}}=I_{y}=I_{y}^{(1)}-I_{y}^{(2)}
\end{gathered}
$$

where the superscripts in round brackets represent quantities of the outer and inner cross sections. In Eqs. (9) and (10), the area moments of inertia of the outer cross-section about the rotating axes $O x$ and $O y$ can be presented in a uniform way regardless of the size of the crack as [8]

$$
\begin{gathered}
I_{x}^{(1)}=\frac{\pi R^{4}}{8}+\frac{\pi R^{4}}{4}\left[(1-\mu)\left(2 \mu^{2}-4 \mu+1\right) \gamma+\sin ^{-1}(1-\mu)\right] \\
I_{y}^{(1)}=\frac{\pi R^{4}}{4}-\frac{\pi R^{4}}{12}\left[(1-\mu)\left(2 \mu^{2}-4 \mu-3\right) \gamma+3 \sin ^{-1} \gamma\right]
\end{gathered}
$$

The quantities of the inner cross-section should be calculated separately. For shallow cracks, we have

$$
I_{x}^{(2)}=I_{y}^{(2)}=\pi r^{2} / 4
$$

For those the crack size is large, the formulas run into

$$
\begin{gathered}
I_{x}^{(2)}=\frac{\pi r^{2}}{4}-2 \int_{-r}^{-(R-h)} \sqrt{r^{2}-y^{2}} y^{2} d y \\
I_{y}^{(2)}=\frac{\pi r^{2}}{4}-2 \int_{0}^{\sqrt{r^{2}-(R-h)^{2}}}\left[\sqrt{r^{2}-x^{2}}-(R-h)\right] x^{2} d x
\end{gathered}
$$

Without loss of generality, if an open crack is located at the $j$ th element, the time-variant local matrix can be obtained by the coordinate transformation [15]

$$
\mathbf{k}_{f}^{j}=\boldsymbol{\psi}^{\mathrm{T}} \mathbf{k}_{r}^{j} \boldsymbol{\psi}
$$

where $\mathbf{k}_{r}^{j}$ is the local stiffness matrix of the Timoshenko beam element established using the area moments in the rotating coordinates $O^{\prime} \overline{x y}$. Its detailed expression is given in Appendix A. Notation $\psi$ is the transformation matrix from the rotating coordinates $O^{\prime} \overline{x y}$ to the fixed coordinates $O^{\prime} \bar{X} \bar{Y}$ or from $O x y$ to $O X Y$, which can be written as 


$$
\boldsymbol{\psi}=\operatorname{diag}\left(\boldsymbol{\psi}_{0}, \boldsymbol{\Psi}_{0}, \boldsymbol{\psi}_{0}, \boldsymbol{\psi}_{0}\right), \boldsymbol{\psi}_{0}=\left[\begin{array}{cc}
\cos (\Omega t) & -\sin (\Omega t) \\
\sin (\Omega t) & \cos (\Omega t)
\end{array}\right]
$$

Combining Eq. (16) and Eqs. (6) (8), the final expression for the local stiffness matrix of the cracked element is given by

$$
\mathbf{k}_{f}^{j}=\mathbf{k}_{1}^{j}+\mathbf{k}_{2}^{j} \cos (2 \Omega t)+\mathbf{k}_{3}^{j} \sin (2 \Omega t)
$$

The elements in the stiffness matrices $\mathbf{k}_{1}^{j}, \mathbf{k}_{2}^{j}$ and $\mathbf{k}_{3}^{j}$ are provided in Appendix B. To this stage, the crack has been fully modeled mathematically. Then the general equations of the rotor system can be derived by an assemblage of all the elements, which yields

$$
\mathbf{M} \ddot{\mathbf{U}}(t)+\hat{\mathbf{C}} \dot{\mathbf{U}}(t)+\left(\mathbf{K}_{1}+\mathbf{K}_{2} \cos (2 \Omega t)+\mathbf{K}_{3} \sin (2 \Omega t)\right) \mathbf{U}(t)=\mathbf{F}_{g}+\mathbf{F}_{1} \cos (\Omega t)+\mathbf{F}_{2} \sin (\Omega t)
$$

where $\ddot{\mathbf{U}}, \dot{\mathbf{U}}$ and $\mathbf{U}$ are the acceleration, velocity and displacement vectors, respectively. The $\mathbf{M}$ represents the global mass of the rotor system. The matrix $\hat{\mathbf{C}}$ includes the damping and gyroscopic terms. The matrix $\mathbf{K}_{1}$ stands for the complete stiffness matrix with the entries at the cracked element being replaced by $\mathbf{k}_{1}^{j}$, and $\mathbf{K}_{2}$ and $\mathbf{K}_{3}$ represent the stiffness matrices which are contributed by $\mathbf{k}_{2}^{j}$ and $\mathbf{k}_{3}^{j}$, respectively, in the damaged element and filled with zeros otherwise. They are of the same size $4(N+1) \times 4(N+1)$ with $N$ being the number of elements. The vector $\mathbf{F}_{g}$ denotes the gravitational force. The unbalance forces are divided into the two vectors $\mathbf{F}_{1}$ and $\mathbf{F}_{2}$, which can be given as

$$
\left\{\begin{array}{l}
\mathbf{F}_{1}=\left[0, \cdots, m_{e} d \Omega^{2} \cos \phi, m_{e} d \Omega^{2} \sin \phi, \cdots, 0\right]^{T} \\
\mathbf{F}_{2}=\left[0, \cdots,-m_{e} d \Omega^{2} \sin \phi, m_{e} d \Omega^{2} \cos \phi, \cdots, 0\right]^{T}
\end{array}\right.
$$

where $\phi$ is the initial unbalance phase. In Eq. (20), the non-zero values are located at node 4.

The HBM can be applied here to derive the periodic harmonic solutions of the dynamic system governed by Eq. (19). To this end, the response of the system is represented by a truncated Fourier expansion, which can be written as

$$
\mathbf{U}(t)=\mathbf{A}_{0}+\sum_{k=1}^{n}\left(\mathbf{A}_{k} \cos (k \Omega t)+\mathbf{B}_{k} \sin (k \Omega t)\right)
$$

where $n$ is the largest number of the harmonics considered. As the gravitational forces and unbalance forces are already in constant term or the first component of the Fourier series, we can substitute Eq. (21) into Eq. (19), which yields a set of algebraic equations (AE) for the unknown coefficients

$$
\mathbf{H}(\mu, \Omega) \Theta=\boldsymbol{\Xi}
$$

with 


$$
\mathbf{H}(\mu, \Omega)=\left[\begin{array}{cccccccccccc}
\mathbf{C}_{0} & \mathbf{0} & \mathbf{0} & \mathbf{C}_{2} & \mathbf{C}_{3} & \mathbf{0} & \mathbf{0} & \mathbf{0} & \mathbf{0} & \cdots & \mathbf{0} & \mathbf{0} \\
\mathbf{0} & \mathbf{C}^{(1)}+\mathbf{C}_{2} & \mathbf{C}_{1}^{(1)}+\mathbf{C}_{3} & \mathbf{0} & \mathbf{0} & \mathbf{C}_{2} & \mathbf{C}_{3} & \mathbf{0} & \mathbf{0} & \cdots & \mathbf{0} & \mathbf{0} \\
\mathbf{0} & -\mathbf{C}_{1}^{(1)}+\mathbf{C}_{3} & \mathbf{C}^{(1)}-\mathbf{C}_{2} & \mathbf{0} & \mathbf{0} & -\mathbf{C}_{3} & \mathbf{C}_{2} & \mathbf{0} & \mathbf{0} & \cdots & \mathbf{0} & \mathbf{0} \\
2 \mathbf{C}_{2} & \mathbf{0} & \mathbf{0} & \mathbf{C}^{(2)} & \mathbf{C}_{1}^{(2)} & \mathbf{0} & \mathbf{0} & \mathbf{C}_{2} & \mathbf{C}_{3} & \ddots & \mathbf{0} & \mathbf{0} \\
2 \mathbf{C}_{3} & \mathbf{0} & \mathbf{0} & -\mathbf{C}_{1}^{(2)} & \mathbf{C}^{(2)} & \mathbf{0} & \mathbf{0} & -\mathbf{C}_{3} & \mathbf{C}_{2} & \ddots & \vdots & \vdots \\
\mathbf{0} & \mathbf{C}_{2} & \mathbf{C}_{3} & \mathbf{0} & \mathbf{0} & \mathbf{C}^{(3)} & \mathbf{C}_{1}^{(3)} & \mathbf{0} & \mathbf{0} & \ddots & \mathbf{0} & \mathbf{0} \\
\mathbf{0} & -\mathbf{C}_{3} & \mathbf{C}_{2} & \mathbf{0} & \mathbf{0} & -\mathbf{C}_{1}^{(3)} & \mathbf{C}^{(3)} & \mathbf{0} & \mathbf{0} & \ddots & \mathbf{C}_{2} & \mathbf{C}_{3} \\
\mathbf{0} & \mathbf{0} & \mathbf{0} & \mathbf{C}_{2} & \mathbf{C}_{3} & \mathbf{0} & \mathbf{0} & \mathbf{C}^{(4)} & \mathbf{C}_{1}^{(4)} & \ddots & -\mathbf{C}_{3} & \mathbf{C}_{2} \\
\mathbf{0} & \mathbf{0} & \mathbf{0} & -\mathbf{C}_{3} & \mathbf{C}_{2} & \mathbf{0} & \mathbf{0} & -\mathbf{C}_{1}^{(4)} & \mathbf{C}^{(4)} & \ddots & \mathbf{0} & \mathbf{0} \\
\vdots & \vdots & \vdots & \ddots & \ddots & \ddots & \ddots & \ddots & \ddots & \ddots & \mathbf{0} & \mathbf{0} \\
\mathbf{0} & \mathbf{0} & \mathbf{0} & \mathbf{0} & \cdots & \mathbf{0} & \mathbf{C}_{2} & \mathbf{C}_{3} & \mathbf{0} & \mathbf{0} & \mathbf{C}^{(n)} & \mathbf{C}_{1}^{(n)} \\
\mathbf{0} & \mathbf{0} & \mathbf{0} & \mathbf{0} & \cdots & \mathbf{0} & -\mathbf{C}_{3} & \mathbf{C}_{2} & \mathbf{0} & \mathbf{0} & -\mathbf{C}_{1}^{(n)} & \mathbf{C}^{(n)}
\end{array}\right]
$$

$$
\begin{gathered}
\Theta=\left[\begin{array}{llllllll}
\mathbf{A}_{0} & \mathbf{A}_{1} & \mathbf{B}_{1} & \mathbf{A}_{2} & \mathbf{B}_{2} & \cdots & \mathbf{A}_{n} & \mathbf{B}_{n}
\end{array}\right] \\
\boldsymbol{\Xi}=\left[\begin{array}{llllllll}
\mathbf{F}_{0} & \mathbf{F}_{1} & \mathbf{F}_{2} & \mathbf{0} & \mathbf{0} & \cdots & \mathbf{0} & \mathbf{0}
\end{array}\right]
\end{gathered}
$$

where $\mathbf{C}_{0}=\mathbf{K}_{1}, \mathbf{C}_{2}=0.5 \mathbf{K}_{2}, \mathbf{C}_{3}=0.5 \mathbf{K}_{3}, \mathbf{C}^{(s)}=\mathbf{K}_{1}-(s \Omega)^{2} \mathbf{M}$ and $\mathbf{C}_{1}^{(s)}=s \Omega \hat{\mathbf{C}}, s=1,2, \ldots, n$. The Fourier coefficients vector $\Theta$ can be easily solved by Eq. (22).

\section{Interval collocation method for uncertain dynamic response bounds estimation}

\subsection{Interval problem of uncertain rotor dynamics}

The deterministic solution procedure is derived in the previous section ignoring the presence of uncertainties. It will be invalid when the parametric uncertainties are taken into consideration since the deterministic model is just a special case. In this subsection, we explain the interval uncertain dynamic problem from a mathematical point of view. First, the interval physical parameters such as uncertain excitation forces can be expressed in interval form as

$$
\left\{\begin{array}{l}
{[\xi]=[\underline{\xi}, \bar{\xi}]=\left[\left[\xi_{1}\right],\left[\xi_{2}\right], \ldots,\left[\xi_{m}\right]\right]} \\
\bar{\xi}=\left[\bar{\xi}_{1}, \bar{\xi}_{2}, \ldots, \bar{\xi}_{m}\right] \\
\underline{\xi}=\left[\underline{\xi}_{1}, \underline{\xi}_{2}, \ldots, \underline{\xi}_{m}\right]
\end{array}\right.
$$

where the square brackets designate an interval variable or vector, the overbar and underline define their upper and lower bounds, respectively. Subscript $m$ is the number of uncertain parameters.

Thus, the matrices in Eq. (19) are no longer deterministic due to uncertainties. For example, uncertain mass unbalance will cause the excitation forces to be uncertain as $\left[\mathbf{F}_{1}\right]$ and $\left[\mathbf{F}_{2}\right]$ while variations in the stiffness of the shaft generate an interval stiffness matrix $[\mathbf{K}]$. For the solution process, Eq. (22) can be rewritten as a set of interval AEs

$$
[\mathbf{H}](\Omega,[\xi])[\Theta]([\xi])=[\boldsymbol{\Xi}]([\xi])
$$


where $[\mathbf{H}]$ and $[\boldsymbol{\Xi}]$ are interval matrices. The interval Fourier coefficient vector $[\Theta]$ needs to be solved for the interval harmonic solutions of the cracked rotor system. The aim here is to determine the interval Fourier coefficients with a good trade-off between calculation accuracy and efficiency. Appropriate UQ methodologies should be introduced to deal with the interval AEs shown in Eq. (27).

\subsection{The URSF based on the Chebyshev collocation points}

As explained previously, the dynamic responses of the cracked rotor are spread out within unknown intervals due to the interval parameter inputs. In this section, we define a non-intrusive continuous function $[f]([\xi])$, termed the URSF, for the response range assuming the uncertain parameters are independent. It is hereafter expressed as $f(\mathbf{x})$ to follow the traditional habits of simple mathematical functions and avoid repeatable usage of the brackets. It is projected to the uncertain parameter vector as

$$
f(\mathbf{x})=[f]([\xi]), \mathbf{x}=\frac{2 \xi^{I}-(\bar{\xi}+\underline{\xi})}{\bar{\xi}-\underline{\xi}}, \xi^{I} \in[\underline{\xi}, \bar{\xi}], \mathbf{x} \in[-1,1]^{m}
$$

The targeted response can be any individual Fourier coefficient or the sum of them denoting the global harmonic response of the system. The following context will show the construction of the explicit expression for the URSF by combination of the polynomial expansion theory and collocation techniques. Without loss of generality, the one-dimensional problem will be addressed in the first place. It should be noted the exact formulation of the URSF is difficult or even impossible to obtain but we can establish an approximated one with satisfactory accuracy. The $p$-order best square polynomial approximation for a continuous function can be expressed as $[48,50]$

$$
f(x) \approx \frac{f_{0}}{2}+\sum_{i=1}^{p} f_{i} T_{i}(x)
$$

where $\left\{f_{i}\right\}$ are the approximation coefficient and $\left\{T_{i}(x)\right\}$ are the first class of the Chebyshev series which can be written as

$$
\left.T_{i}(x)=\cos (i \arccos x)\right), \quad x \in[-1,1]
$$

Their recurrence relationships are

$$
\left\{\begin{array}{l}
T_{0}(x)=1, T_{1}(x)=x \\
T_{i+1}(x)=2 x T_{i}(x)-T_{i-1}(x)
\end{array}\right.
$$

The coefficients $\left\{f_{i}\right\}$ can be calculated by the Mehler integration with Chebyshev collocations as

$$
\left\{\begin{array}{l}
f_{i}=\frac{2}{\pi} \int_{-1}^{1} \frac{f(x) T_{i}(x)}{\sqrt{1-x^{2}}} \mathrm{~d} x=\frac{2}{\lambda} \sum_{k=1}^{\lambda} f\left(x_{k}\right) T_{i}\left(x_{k}\right) \\
x_{k}=\cos \left(\frac{2 k-1}{2 \lambda} \pi\right), k=1,2, \cdots, \lambda
\end{array}\right.
$$


where $\sqrt{1-x^{2}}$ is the weight term, $\lambda$ is the of collocation number which should be at least $p+1$. The collocation points are actually the zeros of $\lambda$-order Chebyshev polynomial. These collocation points are projected on the real uncertain parameter via Eq. (28), then the discrete values of the function being approximated $f\left(x_{k}\right)$ are known readily by applying the deterministic solution procedure with the uncertain parameter taking the values of the projected collocation points. With respect to Eq. (27), it can be expressed as

$$
\left\{\begin{array}{l}
f\left(x_{k}\right)=\left\{\Theta: \mathbf{H}\left(\Omega, \xi_{k}\right) \Theta\left(\xi_{k}\right)=\boldsymbol{\Xi}\left(\xi_{k}\right)\right\} \\
\xi_{k}=\frac{\underline{\xi_{k}}+\bar{\xi}_{k}}{2}+\frac{\bar{\xi}_{k}-\underline{\xi_{k}}}{2} x_{k}
\end{array}\right.
$$

In this situation, Eq. (29) can be further expressed as

$$
f(x) \approx \vartheta \Lambda \mathbf{T}(x)
$$

where

$$
\begin{gathered}
\boldsymbol{\vartheta}=\left[f\left(x_{1}\right), f\left(x_{2}\right), \cdots, f\left(x_{\lambda}\right)\right] \\
\Lambda=\frac{2}{\lambda}\left[\begin{array}{ccccc}
1 / 2 & T_{1}\left(x_{1}\right) & T_{2}\left(x_{1}\right) & \cdots & T_{p}\left(x_{1}\right) \\
1 / 2 & T_{1}\left(x_{2}\right) & T_{2}\left(x_{2}\right) & \cdots & T_{p}\left(x_{2}\right) \\
\vdots & \vdots & \vdots & \ddots & \vdots \\
1 / 2 & T_{1}\left(x_{\lambda}\right) & T_{2}\left(x_{\lambda}\right) & \cdots & T_{p}\left(x_{\lambda}\right)
\end{array}\right] \\
\mathbf{T}(x)=\left[T_{0}(x), T_{1}(x), \cdots, T_{p}(x)\right]^{T}
\end{gathered}
$$

For multi-dimensional cases, the construction procedure for $f(\mathbf{x})$ can be done in a tensor product way. The $m$-dimensional Chebyshev series can be expressed in tensor form [48]

$$
T_{i_{1} i_{2} \cdots i_{m}}(\mathbf{x})=T_{i_{1}}\left(x_{1}\right) T_{i_{2}}\left(x_{2}\right) \cdots T_{i_{m}}\left(x_{m}\right)
$$

Accordingly, the $m$-dimensional $p$-order approximation expression runs into

$$
f(\mathbf{x}) \approx \sum_{i_{1}=0}^{p} \cdots \sum_{i_{m}=0}^{p} \frac{1}{2^{K}} f_{i_{1} i_{2} \cdots i_{m}} T_{i_{1} i_{2} \cdots i_{m}}(\mathbf{x})
$$

where $\kappa$ is the number of zeros in the tensor elements $i_{1} i_{2} \cdots i_{m} . f_{i_{1} i_{2} \cdots i_{m}}$ is the multiple dimensional approximation coefficient and can be solved by the tensor form numerical integration with collocation points as

$$
\left\{\begin{aligned}
f_{i_{1} i_{2} \cdots i_{m}} & =\left(\frac{2}{\pi}\right)^{m} \int_{-1}^{1} \cdots \int_{-1}^{1} \frac{f(\mathbf{x}) T_{i_{i}, \cdots i_{m}}(\mathbf{x})}{\sqrt{1-x_{1}{ }^{2}} \cdots \sqrt{1-x_{m}}} \mathrm{~d} x_{1} \cdots \mathrm{d} x_{m} \\
& \approx\left(\frac{2}{\lambda}\right)^{m} \sum_{j_{1}=1}^{\lambda} \cdots \sum_{j_{m}=1}^{\lambda} f\left(x_{j_{1}}, \ldots, x_{j_{m}}\right) T_{i_{1} i_{2} \cdots i_{m}}\left(x_{j_{1}}, \ldots, x_{j_{m}}\right) \\
x_{j_{k}}= & \cos \left(\frac{2 k-1}{2 \lambda} \pi\right), k=1,2, \cdots, \lambda
\end{aligned}\right.
$$


Similar to Eq. (33), the deterministic solution at a specific collocation point of the multiple uncertain parameters can be expressed as

$$
\left\{\begin{array}{l}
f\left(x_{j_{1}}, \ldots, x_{j_{m}}\right)=f\left(\mathbf{x}_{j_{1}} \cdots j_{m}\right)=\left\{\Theta: \mathbf{H}\left(\Omega, \xi_{j_{1} \cdots j_{m}}\right) \Theta\left(\xi_{j_{1}} \cdots_{j_{m}}\right)=\boldsymbol{\Xi}\left(\boldsymbol{\xi}_{j_{1}} \cdots_{j_{m}}\right)\right\} \\
\xi_{j_{i}}=\frac{\underline{\xi_{i}}+\bar{\xi}_{i}}{2}+\frac{\xi_{i}-\underline{\underline{\xi}}}{2} x_{j_{i}}, \quad i=1,2, \cdots, m
\end{array}\right.
$$

After all the coefficients are prepared, the multi-dimensional URSF expressed in Eq. (39) can be constructed. Combining with Eq. (31), it is finally given in power basis as

$$
f(\mathbf{x}) \approx \sum_{i_{1}=0}^{p} \ldots \sum_{i_{m}=0}^{p} \frac{1}{2^{\kappa}} \tilde{f}_{i_{1} i_{2} \cdots i_{k}} x_{1}^{i_{1}} \ldots x_{m}^{i_{m}}, \mathbf{x} \in[-1,1]^{m}
$$

where $\tilde{f}_{i_{1} i_{2} \cdots i_{k}}$ are the coefficients after rearrangement. To this point, we have built the approximated function for the uncertain response distribution both in single and multiple dimensions. It is a simple mathematical expression in power basis. The bounds of the URSF can be searched by scanning the simple function, which scatters equal-stepped samplings within each variable interval. It will introduce little burden in the numerical computing tools due to the simplicity of the function expression. Most of the computational efforts are devoted to the calculation of the deterministic response values at those collocation points to construct the expansion coefficients.

\section{Numerical simulations and discussion}

In this section, the steady-state vibrations of the cracked rotor will be investigated based on the interval method and the physical parameters given in Table 1. First, the deterministic vibration characteristics will be demonstrated for a shallow crack in order to have a basic understanding of the dynamic behaviors of the system. Then, the specific uncertainties will be explained. In subsection 5.3, case studies will be carried out considering different interval parameters. All the dynamic responses are drawn at the middle span of the rotor (node 9).

\subsection{Crack signatures based on the deterministic parameters}

For the deterministic case, a shallow crack of $\mu=0.375$ is considered and the corresponding global harmonic responses at node 9 are shown in Fig. 3 where six harmonics are retained. As illustrated in Fig. 3, the crack signatures of the subcritical vibrations are significant even for a shallow crack, where the $2 \times$ and $4 \times$ super harmonic responses are dominant while the vibration component for $3 \times$ is observed but weak. Only subcritical amplitudes of the first-order backward critical speed appeared, which can be distinguished from the case of a breathing crack where both the forward and backward subcritical amplitudes are present [15]. Figure 4 shows the vibration amplitudes for the first six harmonic components. It is clear that the last two harmonic components are very weak, which indicates that fourth-order Fourier expansion is sufficient for the rotor under study. The first four harmonic components for $\mu=0.375$ are further given in Fig. 5. We can see that the hollow-shaft rotor is sensitive to the open crack. 


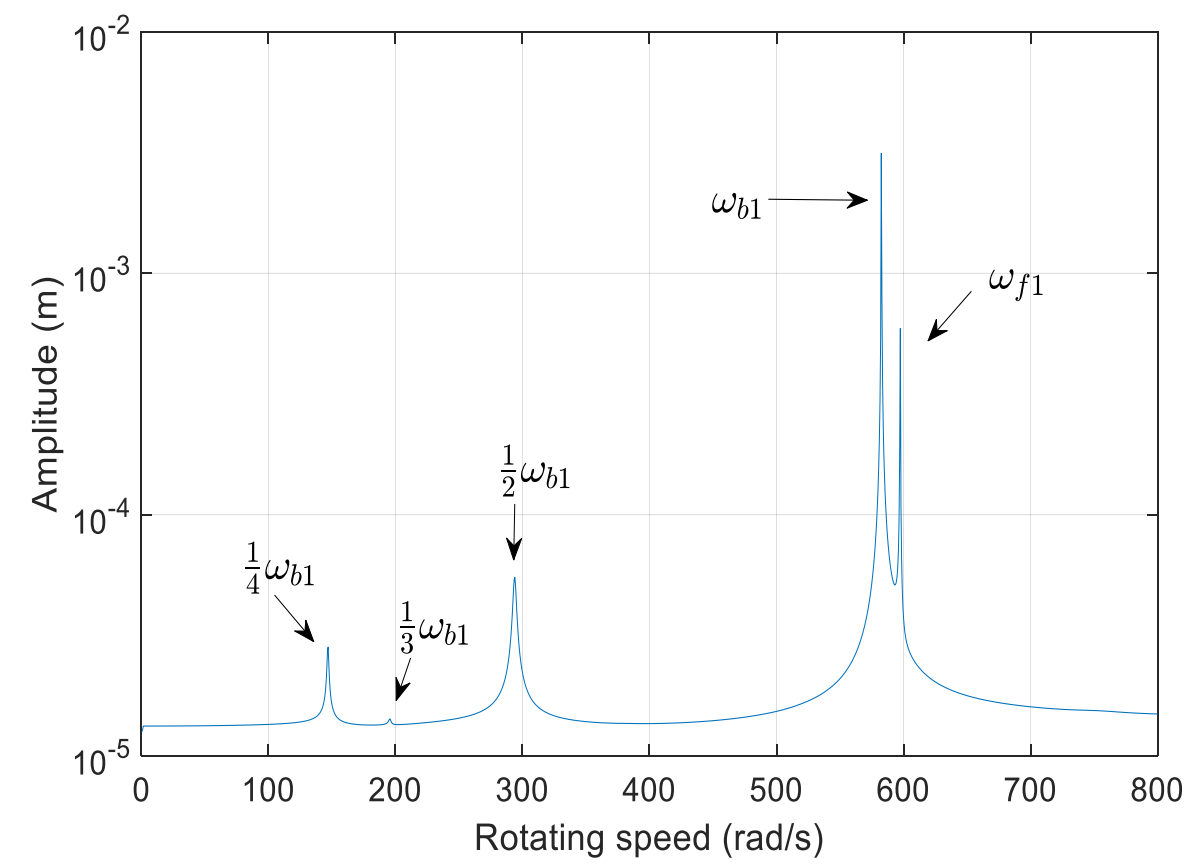

Fig.3. The deterministic global vibration amplitude versus the rotating speed for $\mu=0.375$ when six harmonic components are considered.

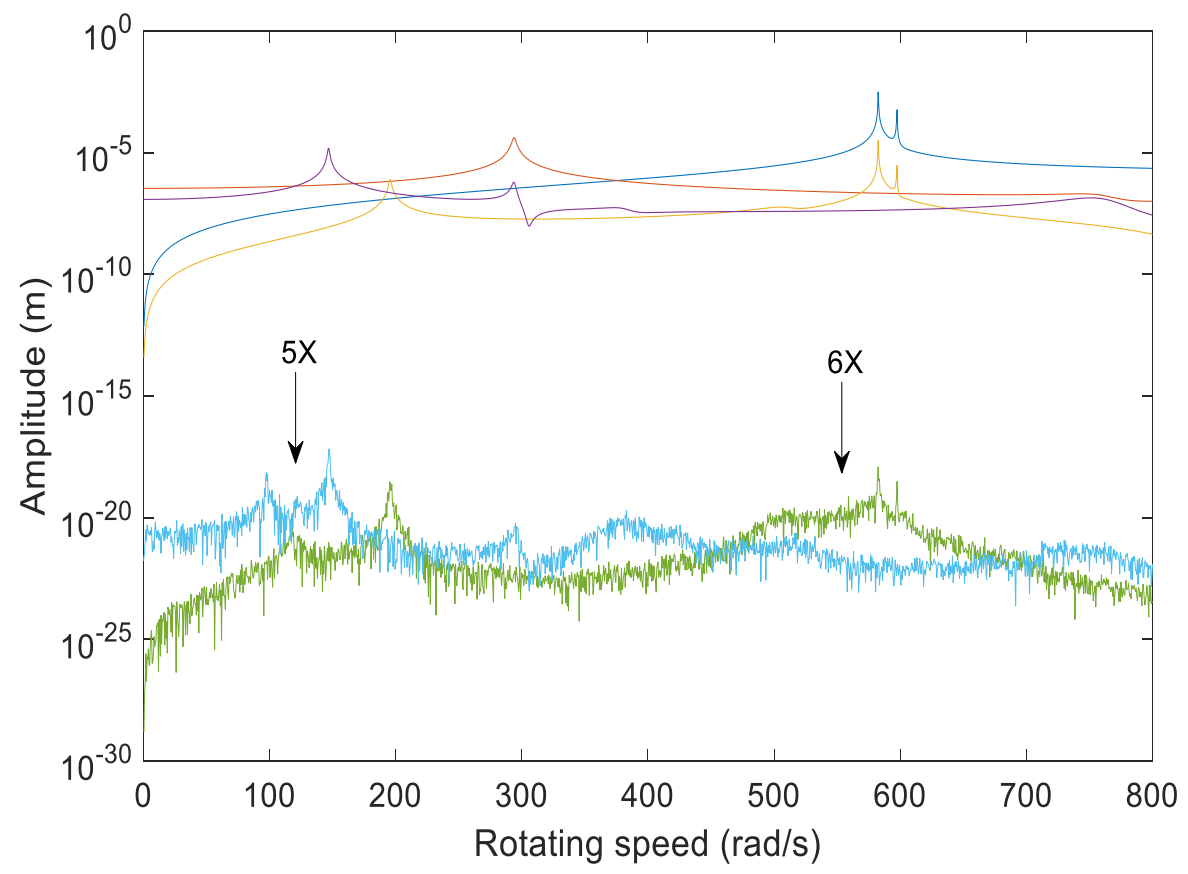

Fig.4. The vibration amplitudes of harmonic components versus the rotating speed for $\mu=0.375$ when six harmonics are considered. 


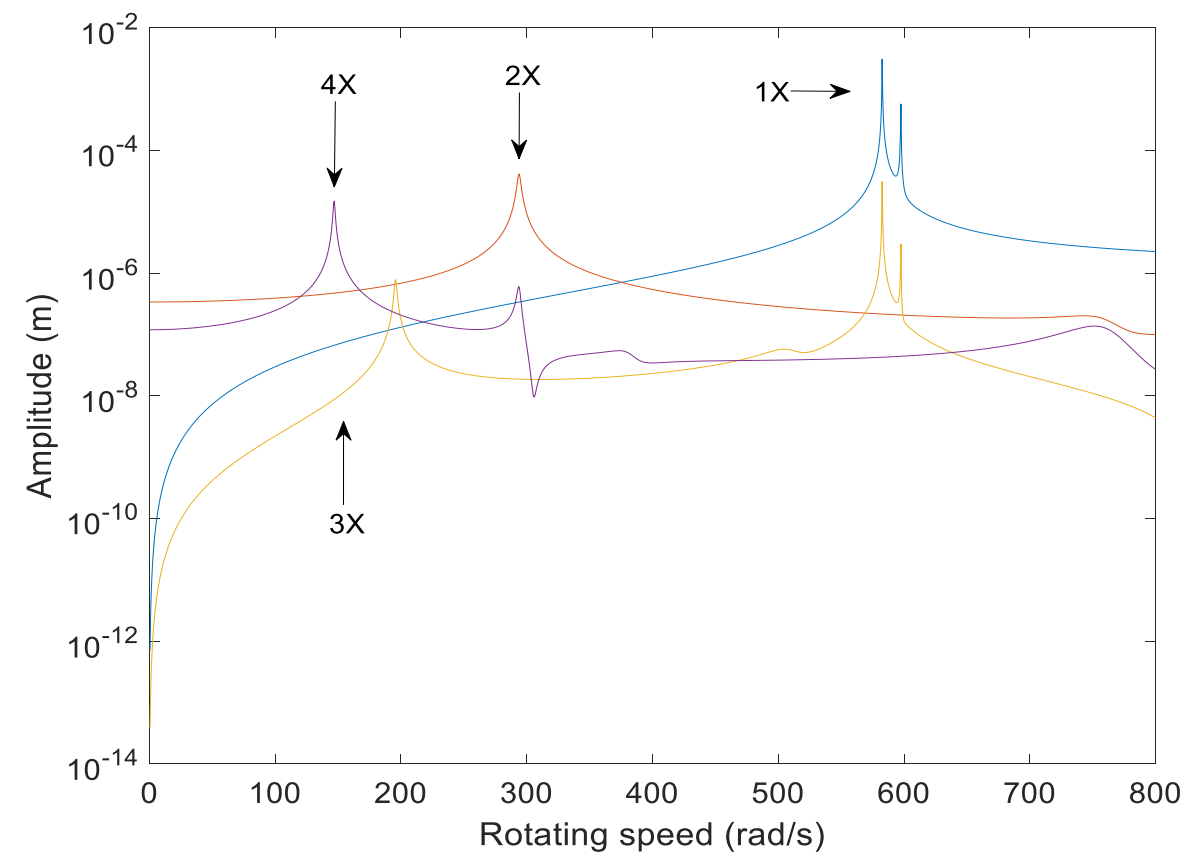

Fig.5. The vibration amplitudes of the first four harmonic components versus the rotating speed for $\mu=0.375$.

\subsection{Uncertainties in the cracked rotor system}

The uncertainties considered in the rotor are described as follows. According to previous statement, the uncertain parameters are all defined as interval variables without any probabilistic models. They can be mathematically expressed by the lower and upper bounds or the nominal values with their respective variation coefficients as well.

First, the stiffness of the shaft is taken as uncertain input, which actually is considered regarding Young's modulus. It can be defined as

$$
[E]=[\underline{E}, \bar{E}]=E\left(1+\beta_{1}[\xi]\right),[\xi]=[-1,1]
$$

where $\beta_{1}$ is the variation coefficient with respect to the nominal value of Young's modulus $E$. For an undetected open crack, the size of it is unknown. During operation, the crack may propagate which is unpredictable and uncertain in a small range of time. Therefore, we treat the non-dimensional crack depth as an uncertain variable and it is given as

$$
[\mu]=[\underline{\mu}, \bar{\mu}]=\mu\left(1+\beta_{2}[\xi]\right)
$$

where $\beta_{2}$ denotes the variation coefficient of the deterministic $\mu$.

Finally, the mass unbalance is taken as interval parameter. It can be time-variant naturally as a result of degradations such as wear. The expression for it is as follows

$$
\left[m_{e}\right]=\left[\underline{m}_{e}, \bar{m}_{e}\right]=m_{e}\left(1+\beta_{3}[\xi]\right)
$$

with $\beta_{3}$ being the variation coefficient of $m_{e}$. 


\subsection{Case investigations}

In this section, the effects of uncertainties on the vibrational characteristics of the cracked rotor are analyzed by the interval procedure proposed previously. The contents are arranged by different sets of uncertain parameters and different level of variation coefficients, which are detailed in Table 2 along with relevant case numbers. Also given in the table are the approximation order $p$ used to build the URSF and the current non-dimensional crack depth. Hereafter, for better legibility in the figures, the vibrations at the subcritical speed areas are focused since they are directly linked to the open crack. In order to validate the accuracy and robustness of the proposed method, comparative results from the MCS, a classical sampling method, are also provided [51]. The MCS is believed to provide solutions with sufficient accuracy using high density samples.

Table 2

Parameter sets for the case investigations.

\begin{tabular}{cccccc}
\hline Case No. & $\beta_{1}$ & $\beta_{2}$ & $\beta_{3}$ & $\mu$ & $p$ \\
\hline 1 & $5 \%$ & - & - & 0.3 & 5 \\
2 & $5 \%$ & - & - & 0.3 & 12 \\
3 & $2.5 \%$ & - & - & 0.6 & 6 \\
4 & $5 \%$ & - & - & 0.6 & 8 \\
5 & - & $5 \%$ & - & 0.3 & 6 \\
6 & - & $2.5 \%$ & - & 0.6 & 8 \\
7 & $2.5 \%$ & $2.5 \%$ & $5 \%$ & 0.3 & 8 \\
8 & $2.5 \%$ & $2.5 \%$ & $5 \%$ & 0.6 & 8 \\
\hline
\end{tabular}

\subsubsection{Cases 1 4: uncertainty in Young's modulus}

In this subsection, the uncertainty in the shaft stiffness is dealt with. The parameter actually treated is Young's modulus, as given in Eq. (43). We consider a shallow crack and a deep one with different variation coefficients for uncertain Young's modulus where all the other parameters of the rotor are kept deterministic. The influences of the URSF order are also investigated. To verify the robustness of the proposed interval method, the results of the MCS are given for comparisons.

Firstly, the global response range of node 9 and the comparisons with the results of the MCS are plotted in Fig. 6 for a shallow crack $\mu=0.3$ with an approximation order 5. In the MCS, 1000 samples are used. From Fig. 6, we can see that the results of the two methods are basically in agreement. Specifically, the bounds estimated by the URSF are identical to that of the MCS in most speed ranges while there 
are unsteady fluctuations in the resonant areas of the $1 / 2$ and $1 / 4$ critical speeds. The cause of this phenomenon is that the responses of the cracked rotor near the resonant peaks are very sensitive to system parameters. They are introduced by numerical errors and instabilities and may not represent the actual dynamic characteristics of the rotor system. However, it can be reduced by increasing the URSF order in view of the fact that only order 5 is used presently. The fluctuations will be weaker and the results of the URSF will envelop closer to the MCS bounds with the increase of surrogate order. Figure 7 gives the corresponding results under the similar conditions as Fig. 6 using the order 12 in the URSF. Extra simulations show that no visible oscillations will be observed when the order is up to 12.The comparison has proved the robustness of the proposed method in the uncertain response bound estimations with a shallow open crack. It should be noted that the efforts of computation are drastically reduced compared to the MCS in which one sample means a complete run of the deterministic case. With the URSF, it takes $\lambda$ (the number of collocations) runs of the FE model. In this paper, the number is set to $\lambda=p+1$ where $p$ is the URSF order. Specifically, the average CPU time for the URSF to accomplish the simulation for Case 2 is $338.62 \mathrm{~s}$ while it will need about 7.69 hours for the MCS to obtain the converged results.

The vibration behaviors of the rotor system subject to Young's modulus uncertainty are influenced significantly, as shown in Fig. 7. Theoretically, the response can take any possible path in the range enveloped by the upper and lower bounds. In resonant regions, the largest amplitudes are less deviated from the deterministic ones (comparing with lower bounds) though they can be large in a close interval around the subcritical speeds. The interesting point is that the upper bounds of the vibration amplitudes are very close to the deterministic values at the exact points of resonances. For the lower bounds, the vibration amplitudes are generally deviated equally to that of the upper bounds but much greater in the resonant areas. It is noticed that the vibration peaks are slightly shifted due to variations in the inherent changes of system critical speeds. For example, in Fig. 7(a) near $150 \mathrm{rad} / \mathrm{s}$, the upper peak is shifted to the left and the lower one to the right compared with the original resonance peak.

To investigate the vibrational characteristics of the rotor with a deep crack, the non-dimensional crack depth is set to 0.6 . Figure 8 shows the interval responses of the middle span of the rotor with 2.5 percent uncertainties in Young's modulus when $\mu=0.6$. The URSF order here is 6 . Increasing the variation degree to $5 \%$, Fig. 9 plots the results with an 8 -order URSF. The global vibration degree is brought up drastically in deep crack cases. In Figs. 8 and 9, the amplitudes at the 1/2 and 1/4 subcritical speeds are large. Larger uncertain degrees lead to wider ranges of the uncertain responses naturally. Here, the effectiveness of the proposed method in estimating uncertain response bounds of the rotor with deep cracks is validated. 

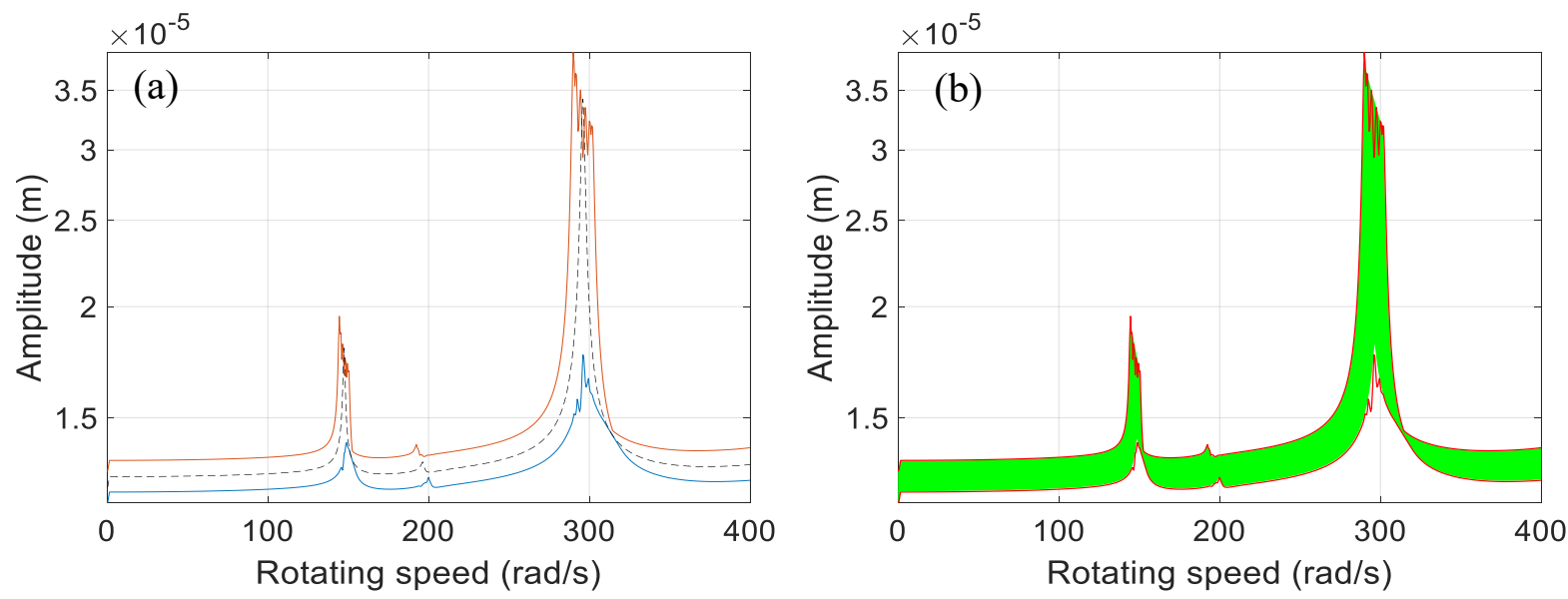

Fig.6. The vibration responses for $\mu=0.3$ with $5 \%$ variations in Young's modulus: (a). Results by the URSF with order 5 (Dashed line - deterministic response; solid lines - bounds by the URSF). (b). Comparisons of results by the URSF (red solid lines) and MCS (green area).
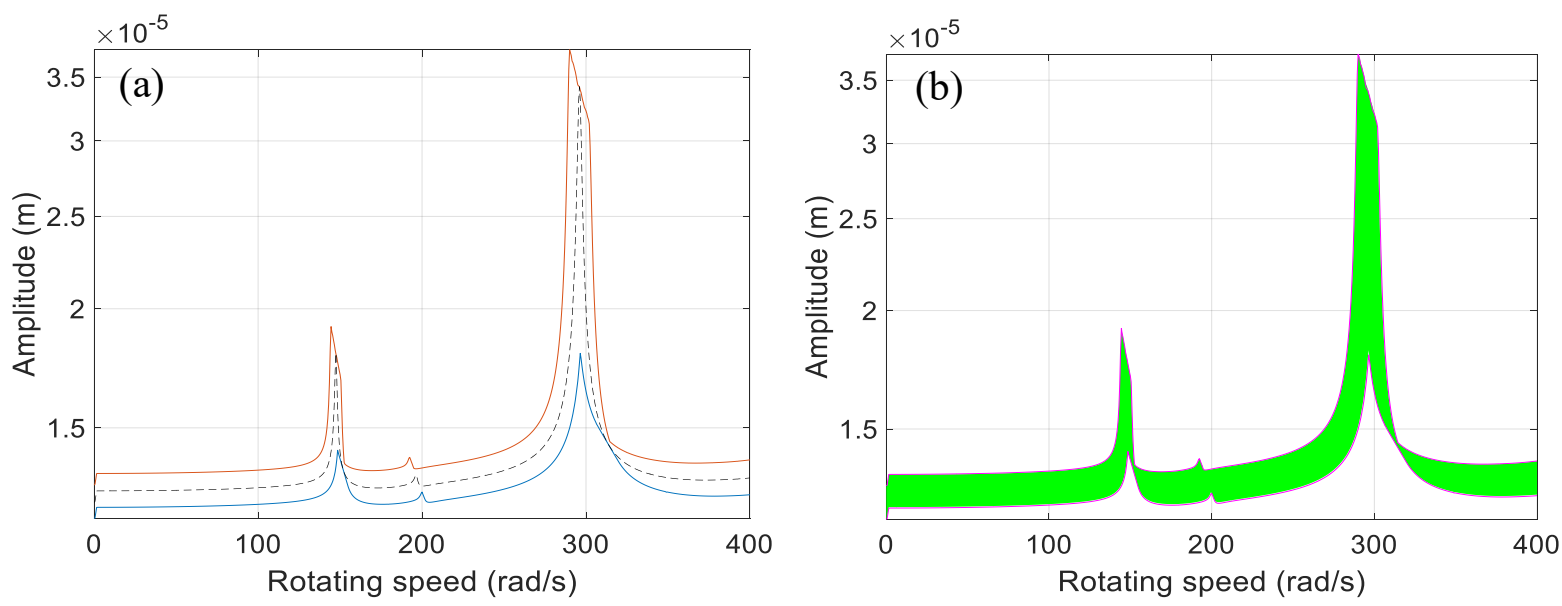

Fig.7. The vibration responses for $\mu=0.3$ with $5 \%$ variations in Young's modulus: (a). Results by the URSF with order 12 (Dashed line-deterministic response; solid lines-bounds by the URSF). (b). Comparisons of results by the URSF (red solid lines) and MCS (green area). 


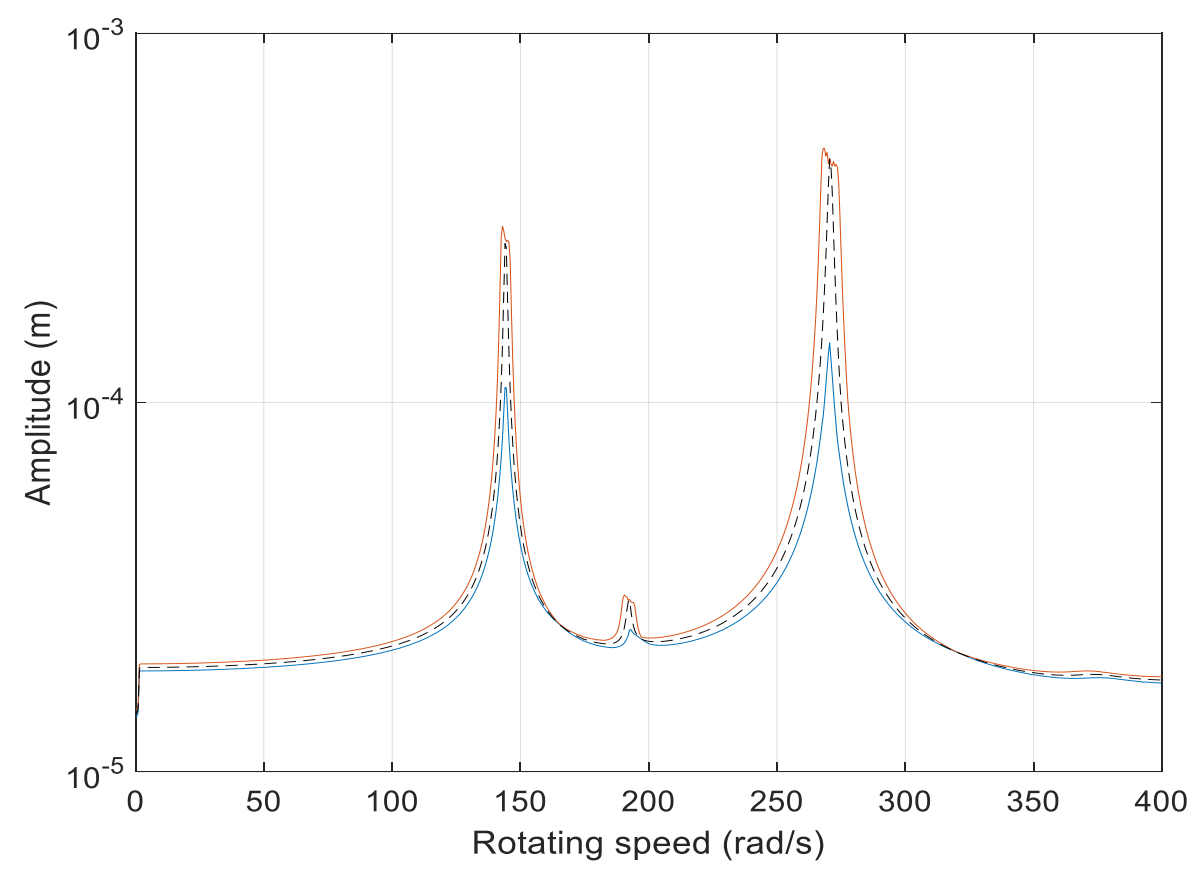

Fig.8. The vibration response evolutions for $\mu=0.6$ with $2.5 \%$ variations in Young's modulus (Dashed line-deterministic response; solid lines — bounds by the 6-order URSF).

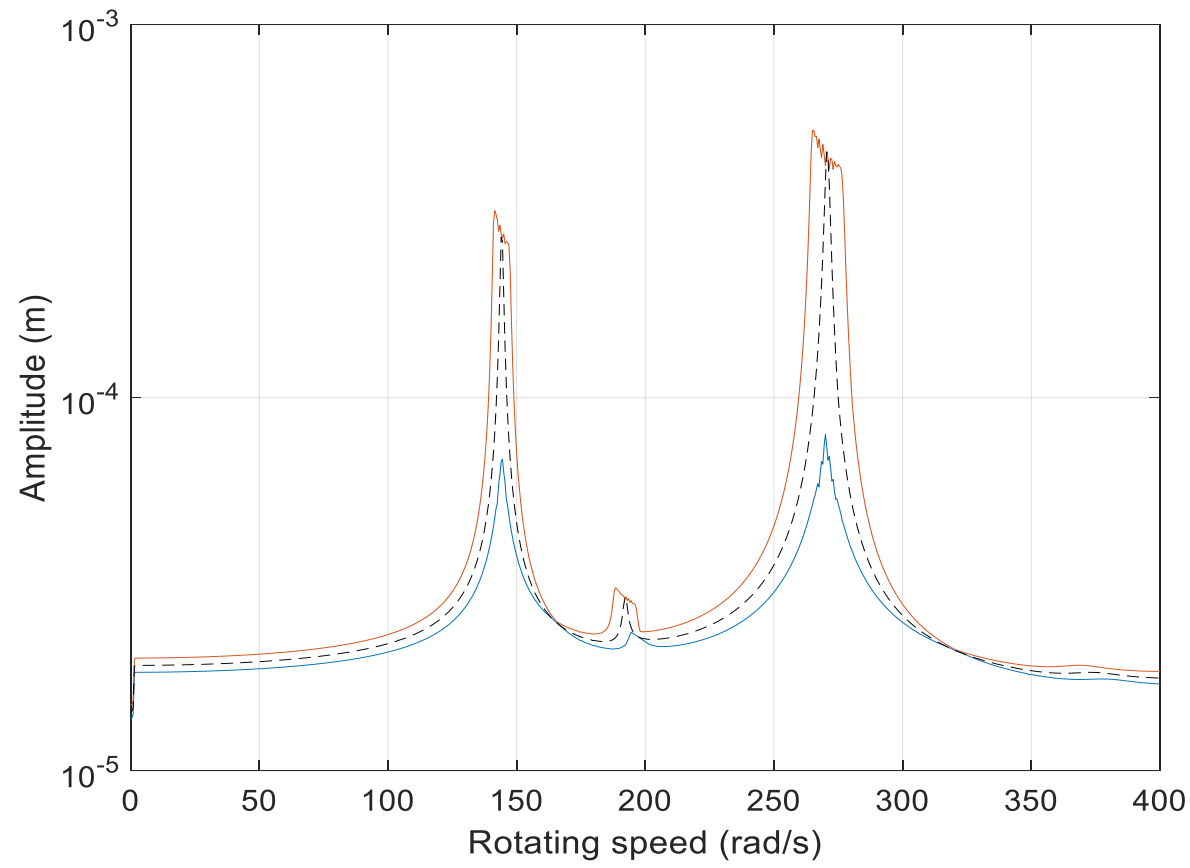

Fig.9. The vibration response evolutions for $\mu=0.6$ with 5\% variations in Young's modulus (Dashed line - deterministic response; solid lines - bounds by the 8-order URSF).

\subsubsection{Cases 5 and 6: uncertainty in the crack depth}

In this part, we focus on the uncertainty in the crack itself. As a hidden fault, the crack needs to be detected with little prior information and it may propagate with time. Therefore, the crack depth or size 
can be uncertain. Considering the uncertainty in the crack depth, the parameter $\mu$ is regarded as an interval variable. Similar to the previous cases, the other parameters are constant except for the crack depth. Follow the same procedure, the uncertain responses can be calculated. Figure 10 illustrates the interval vibration responses of node 9 based on the 6-order URSF when 5\% uncertainty is present in the crack depth for $\mu=0.3$. It can be noted that the dynamic characteristics of the rotor is less sensitive to the crack depth compared to Young's modulus, although offsets are observed. The unique features are that the upper bounds of the vibration amplitudes at the subcritical speeds are increased significantly and the lower bounds are still large. The results of the case where a deep crack $\mu=0.6$ is considered are plotted in Fig. 11. Here, 2.5\% uncertainty is applied and the order is 8 . Figure 12 further gives the accuracy comparisons between the interval method and the MCS with 1000 samples. We can see that, in deep crack condition, the steady state responses of the rotor are quite sensitive. The percentages of deviations of the response bounds are greater than that in the shallow crack case even the degree of uncertainty is only half. Another difference that should be noticed is that the locations of the resonant peaks are less shifted than the former cases 1-4. Slight shifts exist corresponding to the crack depth variations, as the local view in Fig. 11 shows. Here again, it can be seen from Fig. 12 that the bounds estimated by the interval approach proposed are in good agreement with the ranges obtained from the MCS. Weak fluctuations near the resonant peaks are observed. The remarks concluded previously remain valid: the robustness and estimation accuracy of the URSF are clearly demonstrated.

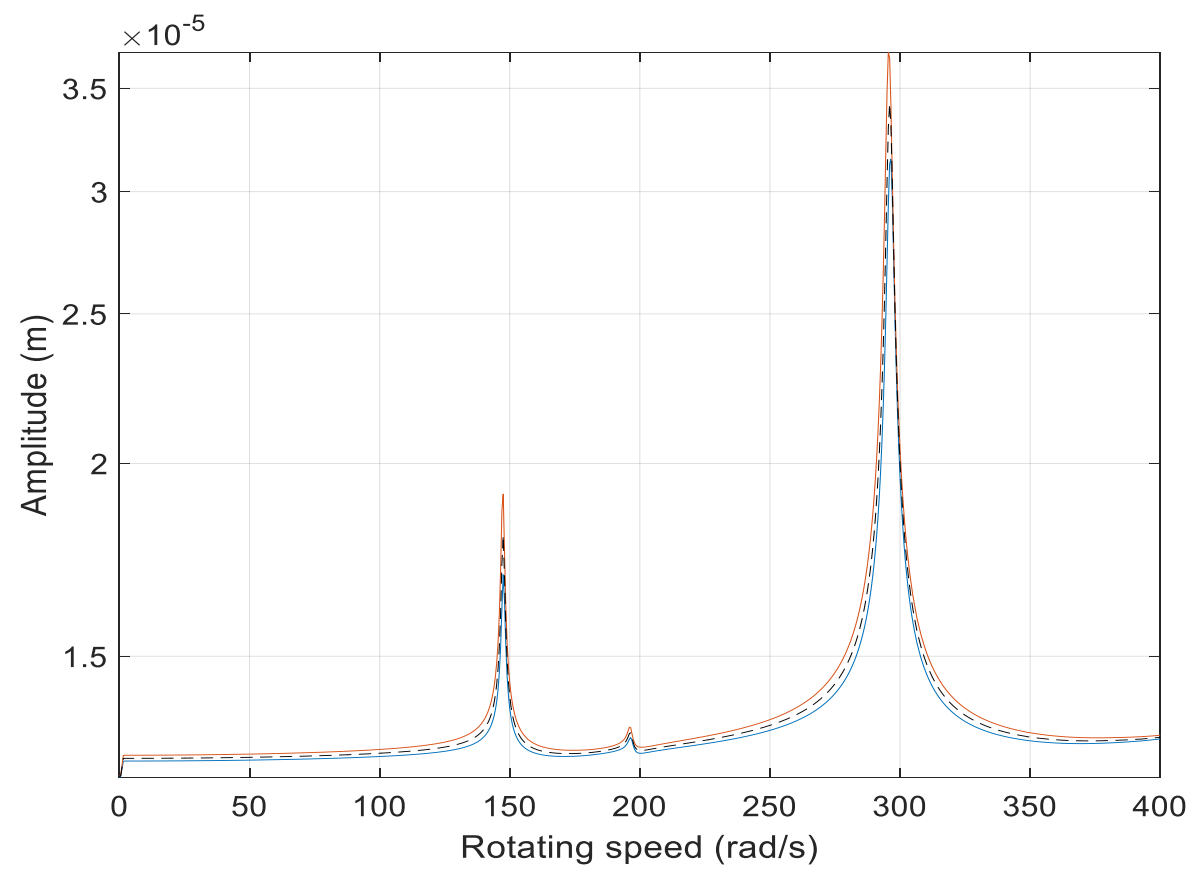

Fig.10. The vibration response evolutions for $\mu=0.3$ with $5 \%$ variations in the crack depth (Dashed line-deterministic response; solid lines-bounds by the 6-order URSF). 


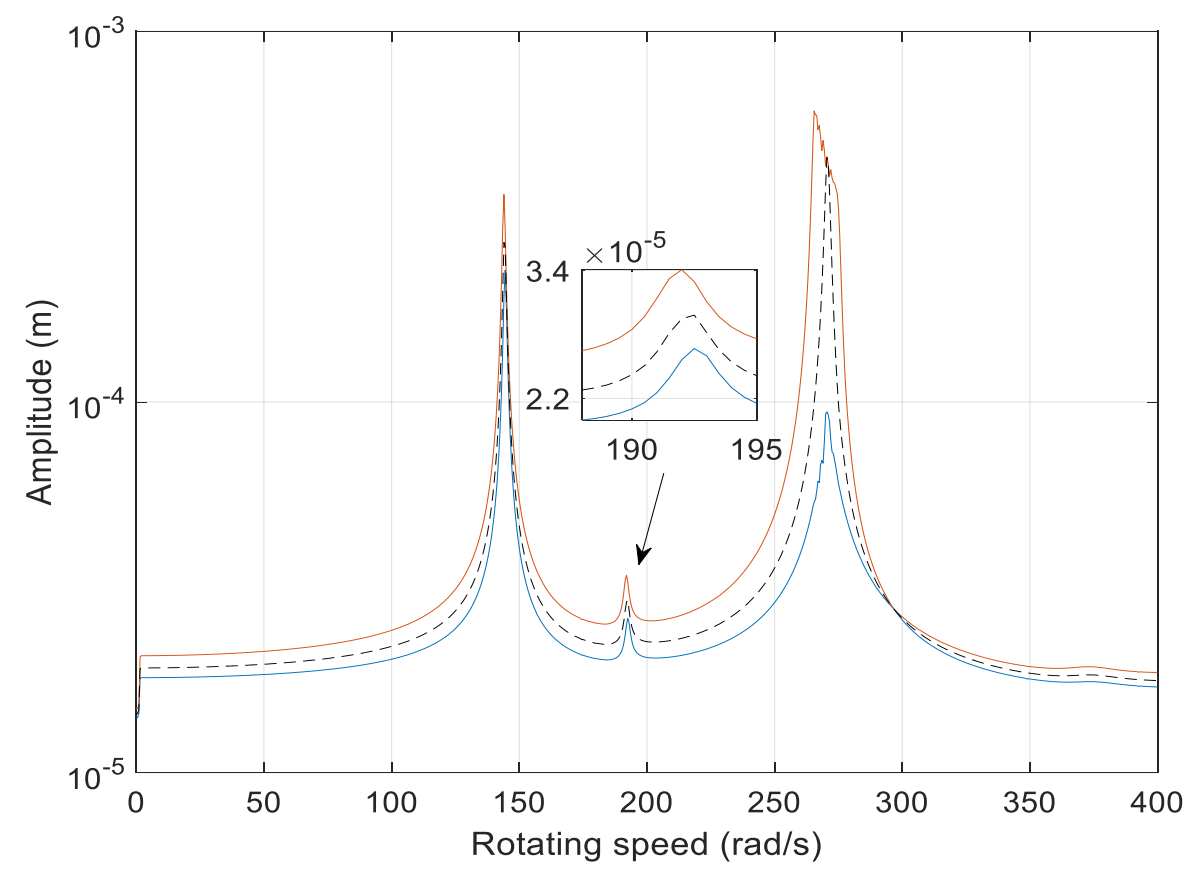

Fig.11. The vibration response evolutions for $\mu=0.6$ with $2.5 \%$ variations in the crack depth (Dashed line - deterministic response; solid lines - bounds by the 8-order URSF).

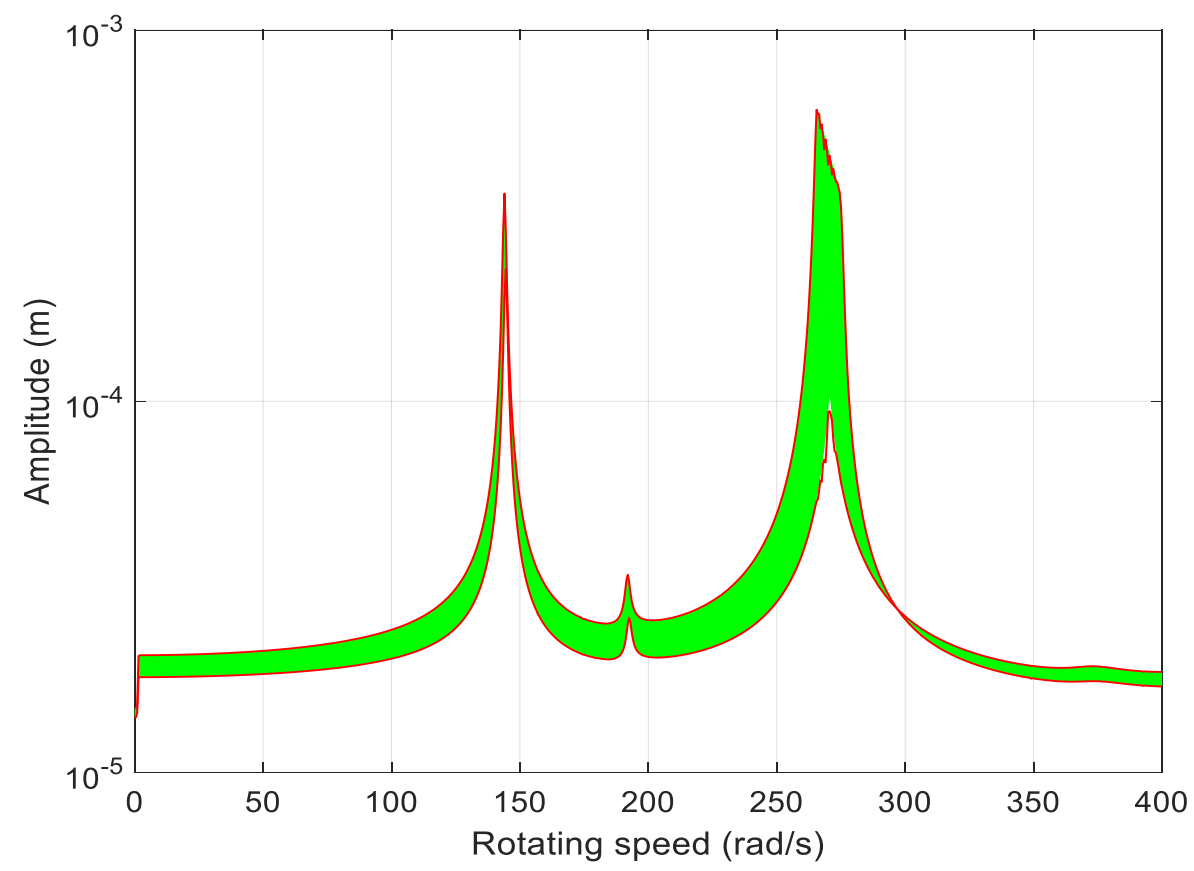

Fig.12. Comparisons of results by the 8-order URSF (red solid lines) and MCS (green area) for $\mu=0.6$ with $2.5 \%$ variations in the crack depth.

\subsubsection{Cases 7 and 8: uncertainties for multiple parameters}

In this subsection, we consider cases with multiple uncertain parameters in the rotor system. Besides the previously studied two uncertainties, $5 \%$ uncertainty in the mass unbalance is added. It should be 
noted that the amount of computation may be high in multi-dimensional cases since the total collocation points are increased significantly. In the MCS, it will be overwhelmed or even impossible in this situation due to the curse of dimension. For example, the overall samples are $10^{6}$ if 100 sampling times is used for each uncertain parameter and the underlying computation cost is huge. Moreover, it is not convincible of the results based on only 100 samples each. Here, we use the scanning method (SCN) for reference to validate the accuracy and robustness of the proposed method. Figure 13 gives the interval results for a shallow case wherein order 8 is used. The comparisons with those obtained from the scanning method are plotted in Fig. 14. For the deep crack case, Figs. 15 and 16 present the response ranges and accuracy validations. Firstly, we can see that good accuracy and robustness of the proposed method can be achieved in multi-dimensional interval problems. The bounds of the two methods are in good accordance and the local fluctuations are weak. From the previous cases, we know that the 8-order URSF is sufficient for problems with $2.5 \%$ uncertainties in the crack depth and Young's modulus. Then, the uncertainties in the mass unbalance have little influence on the accuracy and the unsteady fluctuations in the resonant regions when an 8-order URSF is used. The vibration characteristics of the rotor in the three uncertain parameter cases are different from the previous ones. The vibration amplitudes in the resonant regions are increased significantly and the peak values of the vibration amplitudes at the subcritical speeds are not close to the deterministic ones. Variations in the subcritical speeds are spotted. Comparing the bounds in Figs. 13 and 15, we can find that in deep crack cases the responses are more sensitive to the multi-dimensional uncertainties and the vibration behaviors are influenced significantly. The resonant regions in Fig. 15 are drastically expanded and the peak amplitudes are much higher. In higher dimensional problems, the overall influence of the parametric uncertainties will be even more significant. For the problems with multiple uncertain variables, the sensitivity of each physical parameter may need to be evaluated. Although the quantitative values of the sensitivities should be calculated by a dedicated algorithm such as the Sobol's indices [57, 58], the qualitative assessments can be obtained by simulations with individual uncertainty using the proposed method. The sensitivity is deemed to be high when significant deviations from the deterministic curve are observed in the interval response range. It will be helpful for the engineers to prioritize the critical parameters in design that have significant impacts on the rotor system. 


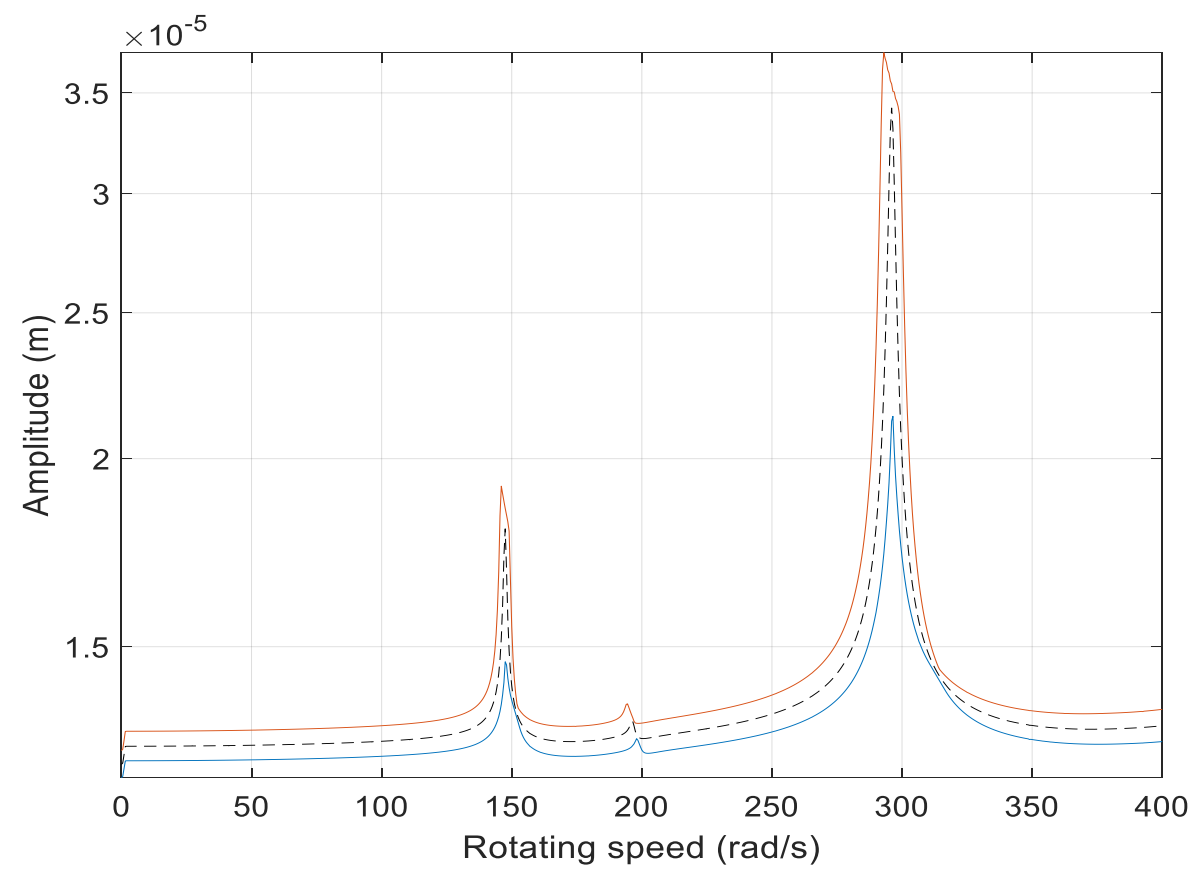

Fig.13. The vibration response evolutions for $\mu=0.3$ with three uncertain parameters (Dashed linedeterministic response; solid lines - bounds by the 8-order URSF).

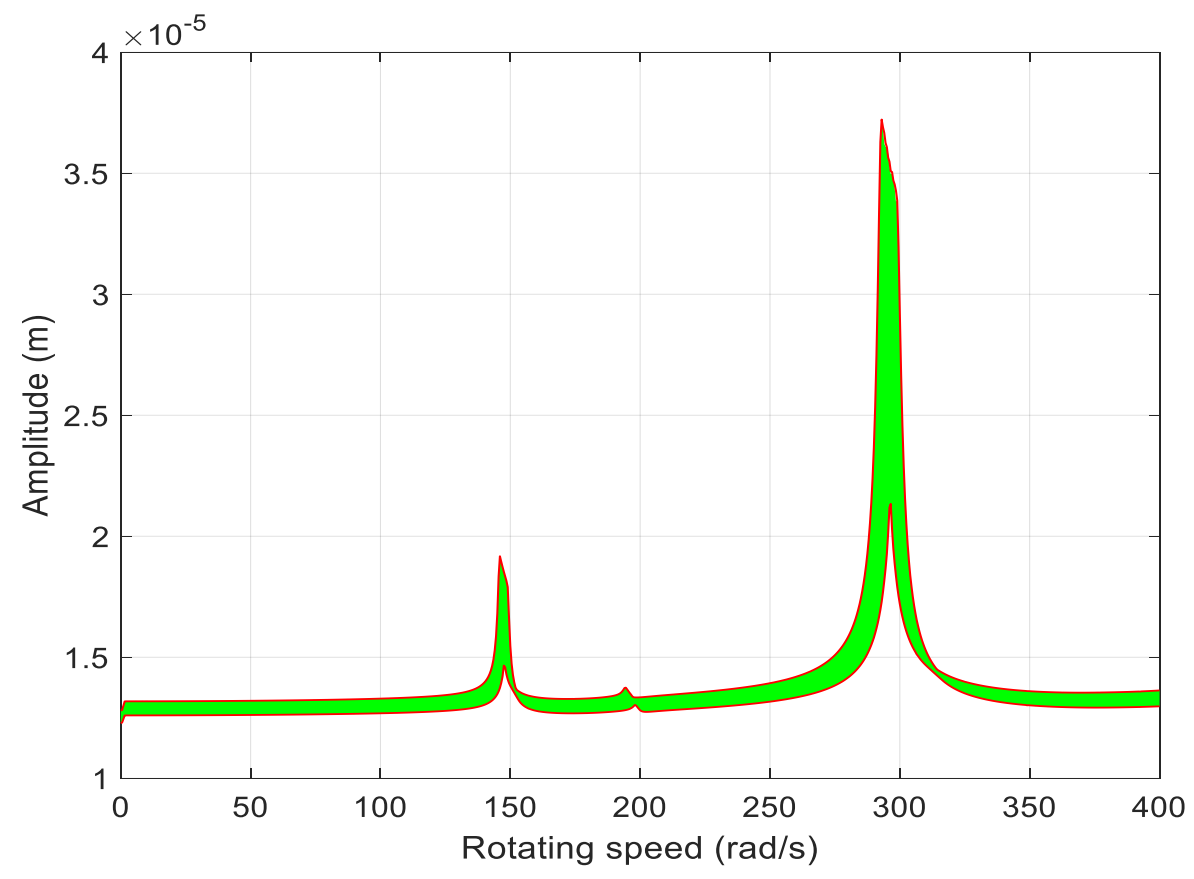

Fig.14. Comparisons of results by the 8-order URSF (red solid lines) and SCN (green area) for $\mu=0.3$ with three uncertain parameters. 


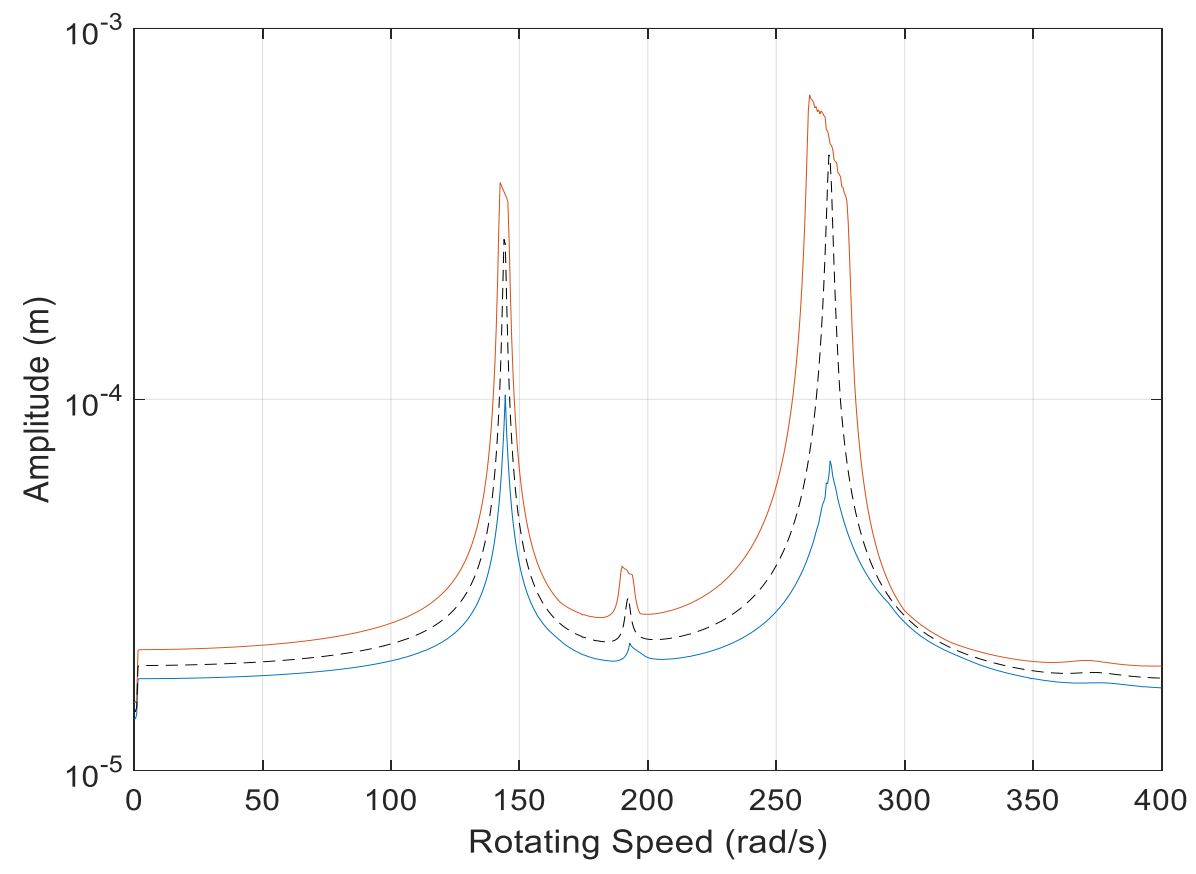

Fig.15. The vibration response evolutions for $\mu=0.6$ with three uncertain parameters (Dashed linedeterministic response; solid lines - bounds by the 8-order URSF).

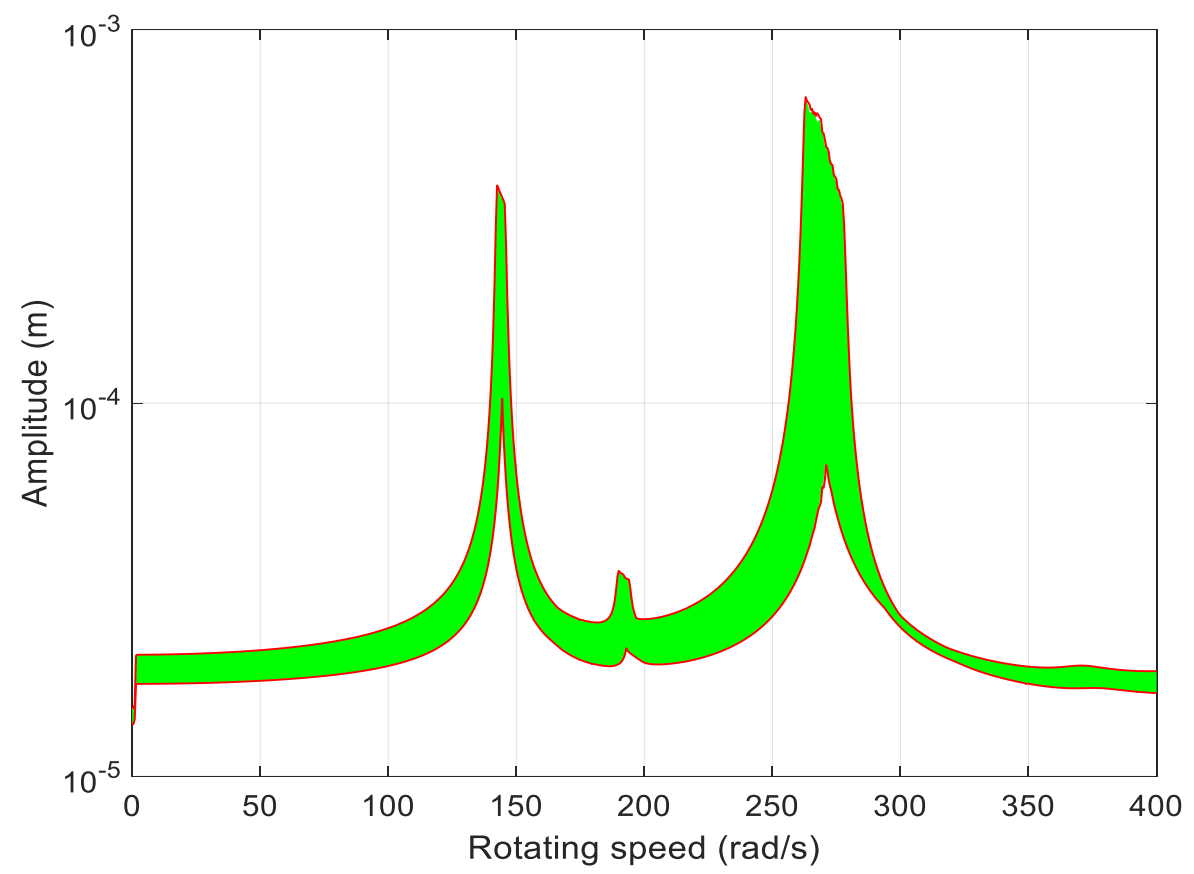

Fig.16. Comparisons of results by the 8-order URSF (red solid lines) and SCN (green area) for $\mu=0.6$ with three uncertain parameters. 


\section{Conclusions}

In this paper, the uncertain response surrogate function (URSF) is developed to investigate the vibration characteristics of a cracked hollow-shaft rotor system with uncertainties. The FEM and the HBM are used to solve the deterministic rotor system. The non-intrusive URSF for the uncertain response range estimation is proposed based on the orthogonal approximation theory using minimum prior information of the uncertainties. The calculation procedure is transparent and easy to follow. Furthermore, it reduces most of the computational efforts in the typical sampling methods. Taking the conventional MCS and the scanning method for references, various numerical simulations have verified the accuracy and robustness of the proposed method both in shallow and deep crack cases under different uncertain coefficients. In particular, it has been found that uncertainties can lead to resonance changes which can cause incorrectness of crack diagnostics method. However, the vibration amplitudes at subcritical speeds can be robust signatures for crack detection.

\section{Acknowledgements}

This work was supported by the National Natural Science Foundation of China [grant number 11272257] and the Fundamental Research Funds for the Central Universities [grant number 3102016ZY016].

\section{Appendix A}

The local stiffness matrix of the cracked element:

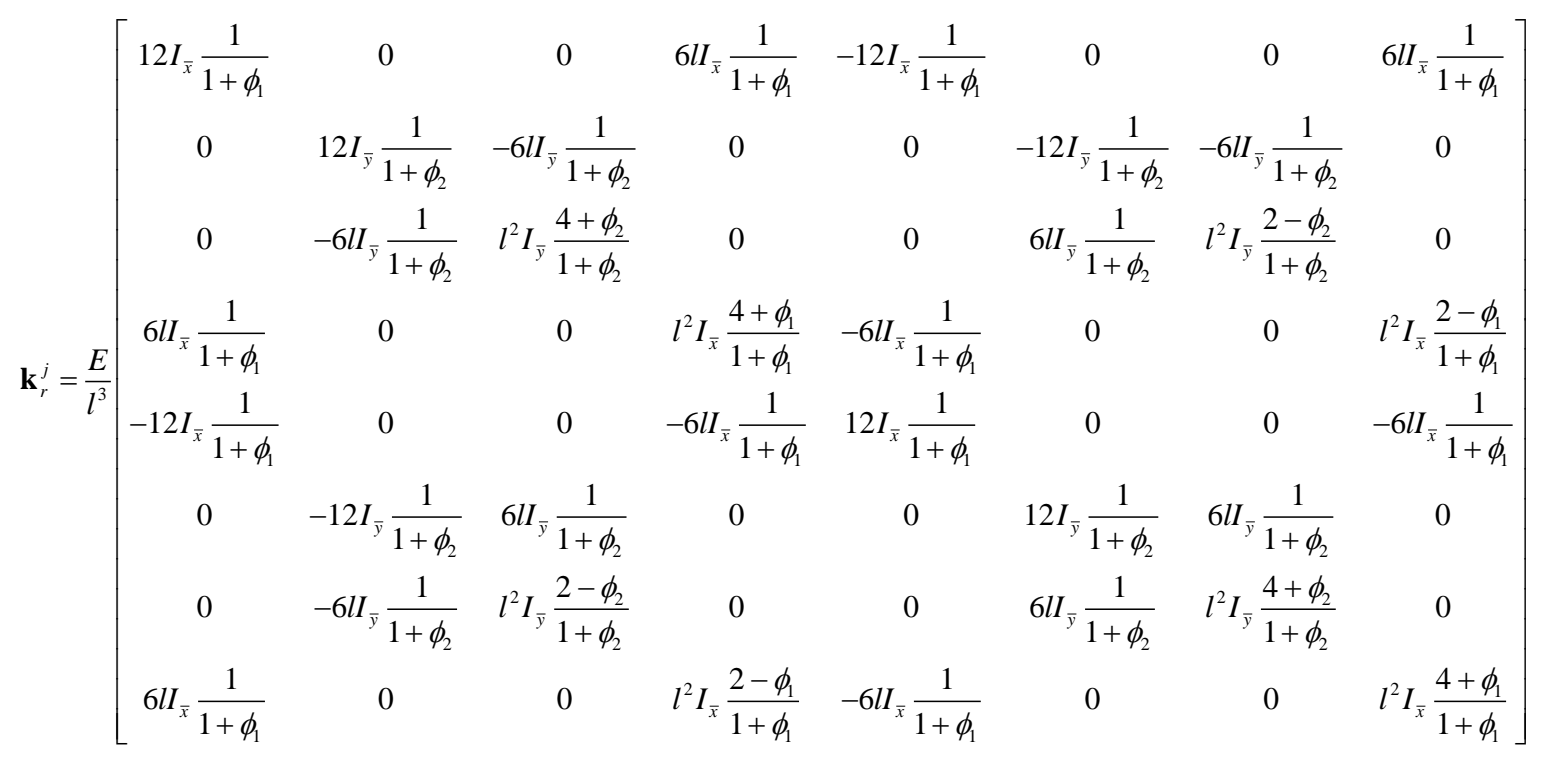

where $\phi_{1}=\frac{12 E I_{\bar{x}}}{\varepsilon A_{\text {rest }} G l^{2}}, \phi_{2}=\frac{12 E I_{\bar{y}}}{\varepsilon A_{\text {rest }} G l^{2}}$ and $G=\frac{E}{2(1+v)}$ represents the shear modulus. The $E, v$, $\varepsilon, A_{\text {rest }}$ and $l$ are Young's modulus, Poisson's ratio, the shear coefficient, the rest area of the crosssection and the length of the cracked shaft element, respectively. 


\section{Appendix B}

The matrices for Eq. (18):

$\mathbf{k}_{1}^{j}=\frac{E}{l^{3}}\left[\begin{array}{cccccccc}12 I_{1} & 0 & 0 & 6 l I_{1} & -12 I_{1} & 0 & 0 & 6 l I_{1} \\ 0 & 12 I_{1} & -6 l I_{1} & 0 & 0 & -12 I_{1} & -6 l I_{1} & 0 \\ 0 & -6 l I_{1} & l^{2} a_{1} & 0 & 0 & 6 l I_{1} & l^{2} b_{1} & 0 \\ 6 l I_{1} & 0 & 0 & l^{2} a_{1} & -6 l I_{1} & 0 & 0 & l^{2} b_{1} \\ -12 I_{1} & 0 & 0 & -6 l I_{1} & 12 I_{1} & 0 & 0 & -6 l I_{1} \\ 0 & -12 I_{1} & 6 l I_{1} & 0 & 0 & 12 I_{1} & 6 l I_{1} & 0 \\ 0 & -6 l I_{1} & l^{2} b_{1} & 0 & 0 & 6 l I_{1} & l^{2} a_{1} & 0 \\ 6 l I_{1} & 0 & 0 & l^{2} b_{1} & -6 l I_{1} & 0 & 0 & l^{2} a_{1}\end{array}\right]$

$\mathbf{k}_{2}^{j}=\frac{E}{l^{3}}\left[\begin{array}{cccccccc}12 I_{2} & 0 & 0 & 6 l I_{2} & -12 I_{1} & 0 & 0 & 6 l I_{2} \\ 0 & 12 I_{3} & -6 l I_{3} & 0 & 0 & -12 I_{3} & -6 l I_{3} & 0 \\ 0 & -6 l I_{3} & l^{2} a_{3} & 0 & 0 & 6 l I_{3} & l^{2} b_{3} & 0 \\ 6 l I_{2} & 0 & 0 & l^{2} a_{2} & -6 l I_{2} & 0 & 0 & l^{2} b_{2} \\ -12 I_{2} & 0 & 0 & -6 l I_{2} & 12 I_{2} & 0 & 0 & -6 l I_{2} \\ 0 & -12 I_{3} & 6 l I_{3} & 0 & 0 & 12 I_{3} & 6 l I_{3} & 0 \\ 0 & -6 l I_{3} & l^{2} b_{3} & 0 & 0 & 6 l I_{3} & l^{2} a_{3} & 0 \\ 6 l I_{2} & 0 & 0 & l^{2} b_{2} & -6 l I_{2} & 0 & 0 & l^{2} a_{2}\end{array}\right]$

$\mathbf{k}_{3}^{j}=\frac{E}{l^{3}}\left[\begin{array}{cccccccc}0 & -12 I_{3} & 6 l I_{3} & 0 & 0 & 12 I_{3} & 6 l I_{3} & 0 \\ -12 I_{3} & 0 & 0 & -6 l I_{3} & 12 I_{3} & 0 & 0 & -6 l I_{3} \\ 6 l I_{3} & 0 & 0 & l^{2} a_{3} & -6 l I_{3} & 0 & 0 & l^{2} b_{3} \\ 0 & -6 l I_{3} & l^{2} a_{3} & 0 & 0 & 6 l I_{3} & l^{2} b_{3} & 0 \\ 0 & 12 I_{3} & -6 l I_{3} & 0 & 0 & -12 I_{3} & -6 l I_{3} & 0 \\ 12 I_{3} & 0 & 0 & 6 l I_{3} & -12 I_{3} & 0 & 0 & 6 l I_{3} \\ 6 l I_{3} & 0 & 0 & l^{2} b_{3} & -6 l I_{3} & 0 & 0 & l^{2} a_{3} \\ 0 & -6 l I_{3} & l^{2} b_{3} & 0 & 0 & 6 l I_{3} & l^{2} a_{3} & 0\end{array}\right]$

where

$I_{1}=\frac{1}{2}\left(\frac{I_{\bar{x}}}{1+\phi_{1}}+\frac{I_{\bar{y}}}{1+\phi_{2}}\right), \quad I_{2}=\frac{1}{2}\left(\frac{I_{\bar{x}}}{1+\phi_{1}}-\frac{I_{\bar{y}}}{1+\phi_{2}}\right), \quad I_{3}=-I_{2}$ 


$$
\begin{aligned}
& a_{1}=\frac{1}{2}\left(\frac{4+\phi_{1}}{1+\phi_{1}} I_{\bar{x}}+\frac{4+\phi_{2}}{1+\phi_{2}} I_{\bar{y}}\right), a_{2}=\frac{1}{2}\left(\frac{4+\phi_{1}}{1+\phi_{1}} I_{\bar{x}}-\frac{4+\phi_{2}}{1+\phi_{2}} I_{\bar{y}}\right), a_{3}=-a_{2} \\
& b_{1}=\frac{1}{2}\left(\frac{2-\phi_{1}}{1+\phi_{1}} I_{\bar{x}}+\frac{2-\phi_{2}}{1+\phi_{2}} I_{\bar{y}}\right), \quad b_{2}=\frac{1}{2}\left(\frac{2-\phi_{1}}{1+\phi_{1}} I_{\bar{x}}-\frac{2-\phi_{2}}{1+\phi_{2}} I_{\bar{y}}\right), b_{3}=-b_{2} .
\end{aligned}
$$

\section{References}

[1] R. Gasch, A survey of the dynamic behaviour of a simple rotating shaft with a transverse crack, J. Sound Vib., 160 (1993) 313-332.

[2] J. Wauer, On the dynamics of cracked rotors: a literature survey, Appl. Mech. Rev., 43 (1990) 13-17.

[3] E. Chipato, A.D. Shaw, M.I. Friswell, Effect of gravity-induced asymmetry on the nonlinear vibration of an overhung rotor. Commun. Nonlinear Sci. Numer. Simulat., 62 (2018) 78-89.

[4] G. Tehrani, M. Dardel, Vibration mitigation of a flexible bladed rotor dynamic system with passive dynamic absorbers, Commun. Nonlinear Sci. Numer. Simulat., 69 (2019) 1-30.

[5] C. Fu, X. Ren, Y. Yang, K. Lu, W. Qin, Steady-state response analysis of cracked rotors with uncertain-but-bounded parameters using a polynomial surrogate method, Commun. Nonlinear Sci. Numer. Simulat., 68 (2019) 240-256.

[6] C. Papadopoulos, A. Dimarogonas, Coupled longitudinal and bending vibrations of a rotating shaft with an open crack, J. Sound Vib., 117 (1987) 81-93.

[7] J.-J. Sinou, A. Lees, The influence of cracks in rotating shafts, J. Sound Vib., 285 (2005) 1015-1037.

[8] H. Ma, J. Zeng, R. Feng, X. Pang, Q. Wang, B. Wen, Review on dynamics of cracked gear systems, Eng. Fail. Anal., 55 (2015) 224-245.

[9] M.A. Al-Shudeifat, E.A. Butcher, New breathing functions for the transverse breathing crack of the cracked rotor system: approach for critical and subcritical harmonic analysis, J. Sound Vib., 330 (2011) 526-544.

[10] J. Zeng, K. Chen, H. Ma, T. Duan, B. Wen, Vibration response analysis of a cracked rotating compressor blade during run-up process, Mech. Syst. Signal Process 118 (2019) 568-583.

[11] C. Guo, J. Yan, W. Yang, Crack detection for a Jeffcott rotor with a transverse crack: An experimental investigation, Mech. Syst. Signal Process 83 (2017) 260-271.

[12] K. Chen, Y. Huangfu, H. Ma, Z. Xu, X. Li, B. Wen, Calculation of mesh stiffness of spur gears considering complex foundation types and crack propagation paths, Mech. Syst. Signal Process 130 (2019) 273-292.

[13] S.K. Singh, R. Tiwari, Detection and localisation of multiple cracks in a shaft system: An experimental investigation, Measurement, 53 (2014) 182-193.

[14] H.T. Liao, Global resonance optimization analysis of nonlinear mechanical systems: Application to the uncertainty quantification problems in rotor dynamics, Commun. Nonlinear Sci. Numer. Simulat., 19 (2014) 19: 3323-3345.

[15] M.A. AL-Shudeifat, On the finite element modeling of the asymmetric cracked rotor, J. Sound Vib., 332 (2013) 2795-2807.

[16] M. Krawczuk, W. Ostachowicz, Modelling and vibration analysis of a cantilever composite beam with a transverse open crack, J. Sound Vib., 183 (1995) 69-89.

[17] M.I. Friswell, J.E. Penny, Crack modeling for structural health monitoring, Struct. Health Monit., 1 (2002) 139-148.

[18] G. Dong, J. Chen, J. Zou, Parameter identification of a rotor with an open crack, Eur. J. Mech. A/Solid., 23 
(2004) 325-333.

[19] N. Dharmaraju, R. Tiwari, S. Talukdar, Identification of an open crack model in a beam based on forceresponse measurements, Comput. Struct., 82 (2004) 167-179.

[20] B. Faverjon, J.-J. Sinou, Identification of an open crack in a beam using an a posteriori error estimator of the frequency response functions with noisy measurements, Eur. J. Mech. A/Solid., 28 (2009) 75-85.

[21] A. Darpe, K. Gupta, A. Chawla, Dynamics of a two-crack rotor, J. Sound Vib., 259 (2003) 649-675.

[22] A. Sekhar, Vibration characteristics of a cracked rotor with two open cracks, J. Sound Vib., 223 (1999) 497 512.

[23] Z. Lu, L. Hou, Y. Chen, C. Sun, Nonlinear response analysis for a dual-rotor system with a breathing transverse crack in the hollow shaft, Nonlinear Dynam., 83 (2016) 169-185.

[24] Z. Lu, Y. Chen, L. Hou, Z. Li, Dynamic characteristics of an open crack in hollow shaft rotor system, J. Aerosp. Power 30 (2015) 422-430.

[25] T. Van der Sande, P. Zegelaar, I. Besselink, H. Nijmeijer, A robust control analysis for a steer-by-wire vehicle with uncertainty on the tyre forces, Vehicle Syst. Dyn., 54 (2016) 1247-1268.

[26] B. Xia, D. Yu, X. Han, C. Jiang, Unified response probability distribution analysis of two hybrid uncertain acoustic fields, Comput. Methods Appl. M., 276 (2014) 20-34.

[27] F. Yamazaki, A. Member, M. Shinozuka, G. Dasgupta, Neumann expansion for stochastic finite element analysis, J. Eng. Mech., 114 (1988) 1335-1354.

[28] C. Soize, R. Ghanem, Physical systems with random uncertainties: chaos representations with arbitrary probability measure, SIAM J. Sci. Comput., 26 (2004) 395-410.

[29] E.H. Koroishi, A.A. Cavalini Jr, A.M. de Lima, V. Steffen Jr, Stochastic modeling of flexible rotors, J. Braz. Soc. Mech. Sci., 34 (2012) 574-583.

[30] T. Ritto, R. Lopez, R. Sampaio, J. Cursi, Robust optimization of a flexible rotor-bearing system using the Campbell diagram, Eng. Optimiz., 43 (2011) 77-96.

[31] J. Didier, J.-J. Sinou, B. Faverjon, Nonlinear vibrations of a mechanical system with non-regular nonlinearities and uncertainties, Commun. Nonlinear Sci. Numer. Simulat., 18 (2013) 3250-3270.

[32] J. Didier, J.-J. Sinou, B. Faverjon, Study of the non-linear dynamic response of a rotor system with faults and uncertainties, J. Sound Vib., 331 (2012) 671-703.

[33] Y. Yang, Q. Wu, Y. Wang, W. Qin, K. Lu, Dynamic characteristics of cracked uncertain hollow-shaft, Mech. Syst. Signal Process 124 (2019) 36-48.

[34] K. Lu, Y. Jin, Y. Chen, Y. Yang, L. Hou, Z. Zhang, Z. Li, C. Fu, Review for order reduction based on proper orthogonal decomposition and outlooks of applications in mechanical systems, Mech. Syst. Signal Process 123 (2019) 264-297.

[35] Z. Li, J. Jiang, Z. Tian, Non-linear vibration of an angular-misaligned rotor system with uncertain parameters, J. Vib. Control 22 (2016) 129-144.

[36] Y. Zuo, J. Wang, W. Ma, Quasimodes instability analysis of uncertain asymmetric rotor system based on 3D solid element model, J. Sound Vib., 390 (2017) 192-204.

[37] J.-J. Sinou, B. Faverjon, The vibration signature of chordal cracks in a rotor system including uncertainties, J. Sound Vib., 331 (2012) 138-154.

[38] J.-J. Sinou, E. Jacquelin, Influence of Polynomial Chaos expansion order on an uncertain asymmetric rotor system response, Mech. Syst. Signal Process 50-51 (2015) 718-731.

[39] R. Murthy, J.C. Tomei, X.Q. Wang, M.P. Mignolet, A. El-Shafei, Nonparametric stochastic modeling of structural uncertainty in rotordynamics: unbalance and balancing aspects, J. Eng. for Gas Turb. Power 136 (2014) 062506. 
[40] S. Zhou, X. Wu, H. Li, Y. Sun, Critical speed analysis of flexible rotor system with stochastic uncertain parameters, J. Vib. Eng. Technol., 5 (2017) 319-328.

[41] S.S. Rao, Y. Qiu, A fuzzy approach for the analysis of rotor-bearing systems with uncertainties, In: ASME 2011 International Design Engineering Technical Conferences \& Computers and Information in Engineering Conference, IDETC/CIE 2011, Washington, DC, USA, 28-31 August 2011, pp. 1033-1044.

[42] J. Wu, Z. Luo, N. Zhang, Y. Zhang, A new interval uncertain optimization method for structures using Chebyshev surrogate models, Comput. Struct., 146 (2015) 185-196.

[43] C. Fu, X. Ren, Y. Yang, K. Lu, Y. Wang, Nonlinear response analysis of a rotor system with a transverse breathing crack under interval uncertainties, Int. J. Nonlin. Mech., 105 (2018) 77-87.

[44] I. Elishakoff, N. Sarlin, Uncertainty quantification based on pillars of experiment, theory, and computation. Part II: Theory and computation, Mech. Syst. Signal Process, 74 (2016) 54-72.

[45] C. Jiang, B. Ni, N. Liu, X. Han, J. Liu, Interval process model and non-random vibration analysis, J. Sound Vib., 373 (2016) 104-131.

[46] D. Moens, M. Hanss, Non-probabilistic finite element analysis for parametric uncertainty treatment in applied mechanics: Recent advances, Finite Elem. Anal. Des., 47 (2011) 4-16.

[47] Z. Qiu, Y. Zheng, L. Wang, Recent developments in the non-probabilistic finite element analysis, J. Harbin Institute Tech. (New Series), 24 (2017) 1-8.

[48] J. Wu, Z. Luo, Y. Zhang, N. Zhang, L. Chen, Interval uncertain method for multibody mechanical systems using Chebyshev inclusion functions, Int. J. Numer. Meth. Eng., 95 (2013) 608-630.

[49] J. Wu, Z. Luo, J. Zheng, C. Jiang, Incremental modeling of a new high-order polynomial surrogate model, Appl. Math. Model., 40 (2016) 4681-4699.

[50] W. Qi, Z. Qiu, A collocation interval analysis method for interval structural parameters and stochastic excitation, Sci. China Phys. Mech., 55 (2012) 66-77.

[51] A.D. Dimarogonas, Interval analysis of vibrating systems, J. Sound Vib., 183 (1995) 739-749.

[52] Y. Ma, Z. Liang, M. Chen, J. Hong, Interval analysis of rotor dynamic response with uncertain parameters, J. Sound Vib., 332 (2013) 3869-3880.

[53] C. Wang, Y. Ma, D. Zhang, J. Hong, Interval analysis on aero-engine rotor system with misalignment, in: ASME Turbo Expo 2015: Turbine Technical Conference and Exposition, 2015, V007AT030A002.

[54] T.N. Shiau, C.H. Kang, D.S. Liu, Interval optimization of rotor-bearing systems with dynamic behavior constraints using an interval genetic algorithm, Struct. Multidiscip. O., 36 (2008) 623-631.

[55] C. Fu, X. Ren, Y. Yang, W. Qin, Dynamic response analysis of an overhung rotor with interval uncertainties, Nonlinear Dynam., 89 (2017) 2115-2124.

[56] C. Fu, X. Ren, Y. Yang, Y. Xia, W. Deng, An interval precise integration method for transient unbalance response analysis of rotor system with uncertainty, Mech. Syst. Signal Process 107 (2018) 137-148.

[57] I.M. Sobol, Global sensitivity indices for nonlinear mathematical models and their Monte Carlo estimates, Math. Comput. Simulat., 55 (2001) 271-280.

[58] A. Trucchia, V. Egorova, G. Pagnini, M.C. Rochoux, On the merits of sparse surrogates for global sensitivity analysis of multi-scale nonlinear problems: Application to turbulence and fire-spotting model in wildland fire simulators, Commun. Nonlinear Sci. Numer. Simulat., 73 (2019) 120-145. 